\title{
LONG-TERM MONITORING OF FLESHY FRUIT AND HARD MAST \\ PRODUCTION AND SEASONAL BIRD DISTRIBUTION AT THE \\ SAVANNAH RIVER SITE, SOUTH CAROLINA
}

\author{
Final Report for: \\ USDA Forest Service \\ Southern Research Station \\ Agreement No. 33-CA-98-275
}

\author{
Submitted to: \\ Dr. John Blake \\ USDA Forest Service \\ Savannah River National Resource Management and Research Institute \\ P.O. Box 710 \\ New Ellenton, SC 29809
}

\begin{abstract}
Submitted by:
Cathryn H. Greenberg, U.S. Forest Service, Bent Creek Experimental Forest, 1577 Brevard Rd., Asheville, NC, 28806.

Douglas J. Levey, Department of Biology, University of Florida, P.O. Box 118525, Gainesville, Florida 32611-8525.
\end{abstract}

June 15, 2009 


\section{Preamble}

This report is divided into three sections, summarizing work we completed on documenting fruit and hard mast production and on the use of fruits by birds at the Savannah River Site (SRS).

\section{Section 1: Fruit and hard mast production in five habitat types at SRS}

This section includes data on fruit and hard mast production, annually. For sake of completeness, we report and analyze the entire dataset from the "fruit project" - not just the data collected during the term of this particular cost-share agreement. In addition to details about which species produce fruit and hard mast in various habitats over time, we also develop yield tables for the most important species. These tables will allow land managers to estimate average levels of production based on inventory data within specific land management units. This section represents the "bread-and-butter" of our project.

\section{Section 2: Comparison of fruit consumption by fledgling versus adult birds at SRS}

This section reports data from an add-on study designed to determine whether fruit is an especially important resource for birds that have recently fledged and are learning to forage on their own. We predicted that these birds would be more reliant than adult birds on fruits, since fruits are relatively easy to find, capture, and consume, compared to the other major food sources (seeds and insects). If this prediction is supported, it would provide evidence of a largely unrecognized role of fruit production in supporting wildlife and could focus management attention on areas of second growth because fruit production is typically highest in those areas (see Section 1). 
Section 3: Relative importance of fruit, seeds, and insects in the diets of overwintering birds at SRS.

This section documents the importance of fruit to overwintering birds at SRS. It breaks new ground in its use of stable isotopes and sophisticated mixing models to quantify consumption of food items that differ in ratios of stable isotopes. It also dovetails with an earlier study by our group on Yellow-rumped Warblers' use of fruit at SRS (Pearson et al. 2003; Oecologia 135:516-523). 


\section{Section 1}

\section{FRUIT AND HARD MAST PRODUCTION IN FIVE HABITAT TYPES AT THE SAVANNAH RIVER SITE, SOUTH CAROLINA}

Abstract: Fleshy fruit is a key food resource for both game and nongame wildlife, and may be especially important for migratory birds during fall and for resident birds and mammals during winter. Hard mast (acorns, hickory and beech nuts) also represent a valuable food resource to numerous game and nongame wildlife species. Land managers need to know the quantities and species of fruit and hard mast produced in different forest types, and how fruit and mast availability vary seasonally. We quantified fleshy fruit and hard mast abundance monthly in $56,0.1$ ha plots in 5 forest types that are common in the Piedmont and coastal plain physiographic regions of the southeastern US. Forest types included upland hardwood forest (UP), bottomland hardwood forest (BH), loblolly pine plantation (LOB), longleaf pine plantation (LLP), and recent clearcuts (CCLL, harvested 1993). Over the 9-year study period, total dry pulp biomass production was highest in upland hardwood and bottomland hardwood habitat types and somewhat lower in both loblolly and longleaf pine plantation. Overall, fruit production (range 38.2-1.0 kg/ha) was highest in recently harvested stands planted with longleaf pine, but decreased with canopy closure. Disturbance-associated species including Rubus cuneifoliis and Rhus copallina, as well as Vaccinium stamineum, produced a large proportion of fruit for the first several years post-harvest. Myrica cerifera was an important fruit producer in clearcuts beginning about 5 years after harvest. Seasonally, fruit availability was highest during late spring through fall. Ilex opaca, Rhus spp., and M. cerifera produced or 
retained fruit during winter months. Most of the major fruit-producing species (and many minor fruit producers, as well) were strongly associated with particular habitat type(s). Hard mast production also differed among the 5 habitat types, with upland hardwood forest producing the vast majority. Important hard mast producers were Quercus nigra (62\% of total) and Q. falcata (10\% of total). Fruit availability at the Savannah River Site differs temporally and spatially due to differences in species composition, fruiting phenology, and the dynamic process of pioneer species' colonization and decline in recently harvested stands. Hard mast production differs temporally and spatially due to differences in the relative abundance of oaks and hickories, as well different temporal patterns of nut production among species. Land managers could enhance fruit availability for many game and nongame species by creating or maintaining young stands within forests, and by retaining a variety of habitat types with their associated fruitproducing plant species. Hard mast production could be enhanced by retaining important upland and bottomland hardwood forest types, and by retaining more oaks in hickories in managed stands such as pine plantation. 


\section{INTRODUCTION}

Fleshy fruit is a key food resource for both game and nongame wildlife (Martin et al. 1951). Also termed soft mast, it is available most of the year, and the vast majority of birds and mammals consume it at least occasionally (Martin et al. 1951, Willson 1986). Because fruit is often abundant, easily captured, and high in energy, it can be a critical resource in the fall for migratory birds (Willson 1986) and in winter for birds when arthropods and other forest food sources are scarce (Skeate 1987, Place and Stiles 1991, McCarty et al. 2002, Greenberg and Forrest 2003, Whitehead 2003, Borgmann et al. 2004, Kwit et al. 2004). Fruit consumption has also been linked to mammalian survival and reproductive success (Rogers 1976, Eiler et al. 1989).

Despite the importance of fruits to wildlife, relatively little is known about spatial or temporal patterns of fruit production in the southeastern United States. Several studies have shown that fruit production is much greater in forest openings caused by natural (e.g., Blake and Hoppes 1986) or silvicultural (e.g., Perry et al. 1999) disturbance than in closed canopy forest. Increased fruit production may continue for several years after disturbance, due to higher light conditions and reduced competition. However, most studies of fruit production are of only limited utility to land managers because they include only a few species, small sample sizes, single seasons, few forest types or age classes, or are too short-term to provide an accurate picture of how fruit availability changes spatially and temporally.

Hard mast (e.g., nuts, such as acorns, hickory nuts, and beech nuts) also represent a valuable food resource to numerous game and nongame wildlife species (Van Dersal, 
1940; Martin et al., 1951). Highly erratic acorn production patterns have a demonstrable influence on the survival and/or recruitment of many wildlife species ranging from black bears (Ursus americanus) (Eiler et al., 1989), to red headed woodpeckers (Melanerpes erythrocephalus) (Koenig and Mumme, 1987) and white-footed mice (Peromyscus leucopus)(Wolff, 1996) populations. Wolff (1996) suggests that acorns function as a "keystone" forest resource by influencing small mammal prey populations.

Because fruit and hard mast are important to wildlife, land managers need information on both short- and long-term changes in fruit production in response to common silvicultural practices such as regeneration (or reproduction) cutting. Specifically, managers need to know how forest management activities affect the quantities and species of fruit produced in different forest types, and how fruit production varies seasonally and as young stands mature.

To optimize utility for land managers, fruit and hard mast production values should be easily applied to existing or target inventory data. For example, estimates of fruit or hard mast yield by trees might include the average number of fruits produced per tree or per $\mathrm{m}^{2}$ basal area (Greenberg and Parresol 2002). Fruit yield by shrubs might be estimated per unit "volume," (mean height x mean percent cover), or another measure that is quickly and easily obtained in the field. All averages of fruit yield should include data from replicated, randomly selected plants (thereby including both fruiting and nonfruiting plants) of specified size classes and species over multiple years (thereby incorporating variability and periodicity in production).

We quantified fruit and hard mast abundance monthly in 5 managed and unmanaged forest types common in the Piedmont and coastal plain physiographic regions 
of southeastern US (upland hardwood, bottomland hardwood, loblolly pine plantation, longleaf pine plantation, and recent clearcuts). In this report we examine spatial and temporal variation in fruit production and composition between the 5 forest types during a 9-year study period. We also developed yield tables for the most important fruitproducing plant species so that land managers can estimate average fruit production based on inventory data within specific land management units.

\section{METHODS}

\section{Study Area}

We conducted our study at the U.S. Department of Energy's Savannah River Site, a National Environmental Research Park located in Aiken and Barnwell Counties, South Carolina, U.S.A. $\left(33^{\circ} 18^{\prime} \mathrm{N}, 81^{\circ} 37^{\prime} \mathrm{W}\right)$. The site lies within the Sandhill and upper Coastal Plain physiographic provinces. Forested areas cover $>80 \%$ of the site (Workman and McLeod 1990). The majority of this area consists of a patchy mosaic of managed longleaf pine (Pinus palustris) and loblolly pine (P. taeda) stands, and to a lesser extent, upland and bottomland hardwood stands (Odum 1991, White and Gaines 2000).

We established a total of fifty-six 0.1 ha plots $(50 \mathrm{~m} \times 20 \mathrm{~m})$ in 56 stands of five structurally and floristically distinct habitat types. These plots were separated by at least $600 \mathrm{~m}$ and were assumed to be independent. Ten plots each were placed in bottomland hardwood (BH), upland hardwood (UH), and stands clearcut in 1993 and planted with longleaf pine seedlings (CCLL). Thirteen plots each were placed in longleaf pine (LL) 
and loblolly pine (LOB) stands that were at least 40 years old. At our site, bottomland hardwoods have a nearly continuous canopy layer of trees (e.g., Nyssa biflora, Magnolia virginiana, Quercus spp.) and canopy-reaching vines (Rhus radicans, Smilax spp.), a well-developed understory stratum (e.g., Ilex spp., Persea borbonia, Vaccinium spp.) and a pronounced ground layer of Sphagnum, woody stems, and herbs (e.g., sedges, Mitchella repens, Arisaema triphyllum). Upland hardwoods are characterized by a well-developed canopy (e.g., Quercus spp., Carya spp., Pinus taeda) and understory (e.g., Cornus florida, Ilex opaca, Vaccinium arboreum), and a relatively sparse ground layer. Although clearcuts initially consisted of short, second-growth vegetation (e.g., Phytolacca americana), by the end of our study they had a "canopy" of densely planted Pinus palustris (maximum height $=6 \mathrm{~m}$ ) and often included a well-developed understory (e.g., Myrica cerifera, R. copallina, Prunus spp.) and a ground layer (e.g., grasses, sedges, Vaccinium stamineum, $R$. toxicodendron, Opuntia compressa). Longleaf and loblolly pine plantation habitats have a sparse canopy layer of pines, a few understory shrubs and trees (e.g., M. cerifera, Quercus spp.), and a sparse ground layer that often includes lowlying vegetation (e.g., V. stamineum, R. toxicodendron) and vines (e.g., Vitus rotundifolia, Smilax spp.). Successional development undoubtedly typified clearcut habitats in our study; however, prescribed fires in pine habitats, treefall gaps, and other natural processes contributed some degree of vegetational change in all study plots.

\section{Fruit Censuses}


We censused all fleshy fruits in each study plot in January from 1995 to December 2003. We define fruit functionally, as a seed-containing structure with a nutritious outer coating (typically pulp) consumed by vertebrates. Methods for estimating the number of fruit on a given plant depended on the species' life-form and the number of fruiting individuals of that species in a plot. For species with stems not obviously joined underground and spatially distinguishable canopies, we tagged all "individuals" (regardless of potential underground connections) and estimated number of fruits on each, except when $>10$ individuals were encountered in a given plot. In those cases, we sampled ten randomly chosen individuals and multiplied the mean number of fruits on them by the total number of fruiting conspecifics to estimate fruit abundance of that species in the plot. When possible, all fruits on a given plant were counted. Otherwise, fruits were counted from portions (e.g., branches) of the plant, and these counts were extrapolated to the entire plant. When more than ten fruiting individuals of a given species were in a study plot, the mean number of fruits per individual from the ten sampled plants was used to estimate the remaining number of uncounted fruits in the plot. For species whose individuals were not clearly distinguishable (e.g., clones of Vaccinium stamineum, $R$. toxicodendron, Mitchella repens), the total number of fruits in a $4 \times 50 \mathrm{~m}$ strip down the center of the plot were counted and multiplied by five to estimate the total number of fruits of that species in the entire plot.

We determined dry biomass of ripe fruit pulp from off-plot samples of ripe fruits (for each species, 10 fruits from each of 10 fruiting individuals; $n=100 /$ species). Half of the samples remained intact (i.e., whole fruit samples), and the other half had all pulp removed (i.e., seed samples). Samples were then placed in a drying oven $\left(55^{\circ} \mathrm{C}\right)$ for 7 
days, and per-fruit ripe fruit pulp dry mass (g) was determined from the difference. We calculated fruit production for each species by multiplying the average dry pulp weight of 1 fruit by the number of fruits counted within a plot, and extrapolating to $\mathrm{g} / \mathrm{ha}$.

We used a 2-way ANOVA with repeated measures to compare total fleshy fruit (g/ha) production and production for each species among treatments, years (1994-2003), and test for treatment $\mathrm{x}$ year interactions. We used the Type III sum of squares and associated mean squares as the error term for treatment effects. We interpreted either a significant treatment effect and (or) treatment $\mathrm{x}$ year interaction effect as evidence of a treatment effect, indicating that changes among years differed among the treatments. Post-hoc tests were performed using a Tukey multiple comparison procedure. Data were natural log-transformed to reduce heteroscedasticity. We considered $P<0.05$ as statistically significant. For each species, the month of highest average fruit production (including ripe, unripe, and damaged fruit) was used for inter-annual comparisons. Total annual biomass was calculated by summing the month of maximum production for each species.

\section{Hard Mast Sampling}

All nut-producing tree species (hickories, oaks, and beech) $\geq 7 \mathrm{~cm}$ were individually tagged on all 56, 0.1 ha plots in 1995 . The number of fully developed nuts was visually estimated annually (1995-2003) during mid-August to mid-September. For small trees we tried to count every nut; for larger trees we often counted nuts on a visible portion of the crown and extrapolated to the entire tree (e.g., Koenig et al. 1994). Hard 
mast traps were placed under a subset of trees to serve as comparison to visual estimates. We report here only the results of visual hard mast estimates; however, preliminary analyses indicate that visual estimates are highly correlated with, but lower than production estimates based on hard mast trap data. Therefore our visual estimate data are likely conservative estimates of hard mast production in the five habitat types at the Savannah River Site.

\section{Yield Tables}

During 2001 - 2002 we gathered the additional plant inventory data that was necessary to develop fruit production capability models to quantify average annual fruit and hard mast production on a per-plant (or per unit volume for clonal species). Specifically, we (1) quantified the number (e.g., trees $\geq 2.4 \mathrm{~cm} \mathrm{dbh}$ ) or volume (percent

cover $\mathrm{x}$ mean height $\left.\left(\mathrm{m}^{3}\right)\right)$ (e.g., clonal species) of all individuals of fleshy fruit producing species within plots. Prior, our data included only plants that had fruited - we needed to know the number of plants that had never fruited in order to get accurate estimates of average fruit production per plant. Because the abundance of plants changes over time, we used data only from 1999-2003 (about 2 years before and after the inventory) to calculate average fruit yield per plant. Additionally, we omitted plots that had been recently burned, and included select species (C. florida, I. opaca, M. cerifera, and $V$. stamineum) that were abundant, generally not ephemeral, and important fruit producers. We calculated average fruit yield by summing total fruit production and plant abundance (number of individuals or volume) across all plots, dividing total fruit by the 
number (or volume) of individual plants, and averaging production of (1) known fruiters (e.g., tagged) and (2) the total number of individuals (e.g, tagged + untagged) over the five years (1999-2003).

\section{RESULTS AND DISCUSSION}

\section{Fleshy Fruit Production}

A total of 60 species produced a total of $242 \mathrm{~kg}$ dry edible pulp and 4,373,662 individual fruits within the 56, 0.1 ha study plots at the Savannah River Site during the 9year study period (Table 1). Overall, total fruit biomass ( $\mathrm{g} / \mathrm{ha}$ ) production was highest in CCLL, followed by UH and BH, then LOB, and least in LL (Table 1; Figs. 1,2). Annual dry pulp biomass production varied among years in all 5 habitat types, but was most variable in CCLL. Production ranged from $1.9 \pm 0.3$ to $10.8 \pm 2.1 \mathrm{~kg} / \mathrm{ha}$ in $\mathrm{UH}$; from $1.0 \pm 0.1$ to $11.2 \pm 4.2 \mathrm{~kg} / \mathrm{ha}$ in $\mathrm{BH}$; from $0.8 \pm 0.1$ to $8.6 \pm 2.9$ in $\mathrm{LOB}$; from $0.4 \pm 0.1$ to 13.5 \pm 6.3 in LL, and from $1.0 \pm 0.3$ to $38.2 \pm 16.2$ in CCLL (Table 1; Figs. 1,2).

Several studies show that fruit production is much greater in forest openings caused by natural disturbance (Thompson and Willson 1978, Blake and Hoppes 1986, Levey 1990) or by silvicultural disturbance such as harvesting (Halls and Alcaniz 1968, Johnson and Landers 1978, Stransky and Halls 1980, Campo and Hurst 1980, Stransky and Roese 1984, Perry et al. 1999, Mitchell and Powell 2003) compared to closed canopy conditions. Abundant light, soil disturbance, and reduced competition created by reductions in tree basal area provide optimal conditions for fruit production by many species and for colonization by disturbance-associated species such as Phytolacca 
americana, Rhus copallina, and Rubus spp. that are prolific fruit producers. In the Ouachita mountains of Arkansas and Oklahoma, fruit production was negatively correlated with stand basal area 3 and 5 years post-harvest (Perry et al. 1999).

A few species including Vaccinium stamineum (44\% biomass), Vitis rotundifolia (11\% biomass), Cornus florida (9\%), Ilex opaca (7\%), Rubus (4\%), and Nyssa biflora (4\%) dominated fruit biomass production (Table 1). Other species produced prolific numbers of fruits (e.g., dominated numerically), including Rhus copallina (25\%) M. cerifera (19\%), and Rhus toxicodendron (7\%). However, the relative importance of important fruit-producing species differed among the habitat types (Table 1; Fig. 3). For example, Cornus florida was a major fruit producer in $\mathrm{UH}$, but also contributed to total fruit production in $\mathrm{BH}, \mathrm{LOB}$, and LL habitat types. Ilex opaca was a major fruit producer in $\mathrm{BH}$, but also contributed to total fruit production in UH. Nyssa biflora was an important fruit producer but only in $\mathrm{BH}$. Rhus toxicodendron produced the most fruit in LOB, LL, and CCLL. Several species, including M. cerifera, O. compressa, $R$. copallina, $R$. cuneifolius, and $V$. stamineum produced the most fruit in the CCLL habitat type (Table 1; Fig. 3). Several other fruit-producing species were associated with specific habitat types. For example, Arisaema triphyllum, Ilex glabra, Magnolia virginiana, Mitchella repens, Persea borbonia, Rhus radicans, Smilax laurifolia, Vaccinium corymbosum, and Vaccinium elliottii all produced the most fruit in $\mathrm{BH}$. Species that produced relatively minor amounts of fruit biomass nonetheless contributed cumulatively to differences in total fruit production among habitats. Further, the importance of each fruit species is difficult to evaluate, as any given species may be uncommon in the diets of most animal species, yet could be critical to a few. Differences 
in the distribution of important fruit-producing plant species among the 5 habitat types at the SRS affected the spatial availability of total fruit and different fruit species.

Fruit production also varied temporally (Table 1; Figs. 1, 2, 3). Declines in total fruit production in clearcuts (Figs. 1,2) were largely due to decreased production by important fruit-producing disturbance-associated species such as Rubus spp. and Rhus copallina as the stands matured (Table 1; Fig. 3). Vaccinium stamineum fruit production also declined in CCLL as stands matured (Fig. 3), likely due to decreased light availability with increasing canopy closure. Perry et al. (1999) reported similar interannual patterns of fruit production, with pokeweed fruit comprising the majority of total biomass in young regenerated stands for the first few years but disappearing by the fifth year, with blackberry becoming the dominant producer during the fifth year post-harvest (Perry et al. 1999).

Fruit availability also varied seasonally (Table 2; Fig. 2). Generally, fruit production was highest from late spring through fall (Fig. 2). Fruit production was dominated by important fruit-producing species such as Vaccinium stamineum and Rubus spp. (both dominant in CCLL) in early summer, and by C. florida (dominant in UH) in the fall. However, several important fruit-producing species, including I. opaca (dominant in BH), M. cerifera, and R. copallina (both dominant in CCLL), reached peak production during winter. Hence peak fruit availability differed both spatially and temporally among the 5 habitat types due to differences in species composition, fruiting phenology, and the dynamic process of colonization and decline of "pioneer" plant species in CCLL (Figs. 2, 3). 
Our estimates of total fruit production were generally lower than estimates in other studies. Perry et al. (1999) estimated $80-100 \mathrm{~kg} / \mathrm{ha}$ dry fruit biomass in regenerated and clearcut pine-hardwood stands in the Ouachita mountains of Arkansas and Oklahoma 5 years after harvest, whereas our estimates were $\leq 38.2 \mathrm{~kg} / \mathrm{ha}$ in young regenerated stands within the first few years post-harvest, and only $1.0-2.4 \mathrm{~kg} / \mathrm{ha} 9$ and 10 years post-harvest. This large discrepancy is likely in part because our estimates were based on dry edible pulp only, whereas theirs (and most others) are based on whole dry fruits, including seeds. In the southern Appalachians Greenberg et al. (2007), following similar methodologies to the current study, fruit production estimates ranged $0.5-2.0$ $\mathrm{kg} / \mathrm{ha}$ in mature hardwood forest, and up to $16.0 \mathrm{~kg} / \mathrm{ha} 5$ years after harvest. We believe that reporting dry edible pulp provides a more practical estimate of fruit production for land managers, as seeds of fleshy fruit are not generally digested by frugivorous wildlife (seed predators being the exception).

Geographic and site differences may also have contributed to differences in total fruit production because these factors influence the presence and relative abundance of fruit-producing species. For example, at the Savannah River Site (SRS), located between the Piedmont and coastal plain in South Carolina, V. stamineum produced an average of $6.8 \mathrm{~kg}$ dry edible pulp/ha in clearcuts during the first 10 years after harvest, whereas it was a relatively minor producer in the southern Appalachians $(\leq 0.4 \mathrm{~kg} / \mathrm{ha}$ per year in UHM). Dogwood produced an average of $2.0 \mathrm{~kg}$ dry pulp/ha in upland hardwood forest at the SRS during the 9-year study period, but $<0.1 \mathrm{~kg}$ dry pulp/ha per year in southern Appalachian upland hardwood forest. American holly, which retains fruit during winter, produced an average of $1.7 \mathrm{~kg}$ dry pulp/ha in upland and bottomland hardwoods at the 
SRS during the 9-year study period, but a maximum of about $0.01 \mathrm{~kg}$ dry pulp/ha in a somewhat equivalent habitat type in the southern Appalachians.

In our study, spatial variation in fruit production was influenced primarily by habitat type, and likely also by forest management for pine plantations where a high density of pines are planted and competition from other species is reduced through management activities. Stand age was also an important influence on total fruit production in the CCLL habitat type.

\section{Fruit Yield Tables}

The average number of fruits produced per individual plant unit is presented in Table 3. Using this information, land managers can estimate average fruit production within specific land management units by applying mean values to inventory data (e.g., number of individuals or $\mathrm{m}^{3}$ volume, for clonal shrubs).

\section{Hard Mast Production}

A total of 11 nut-producing trees including 11 oak species, 2 hickory species, and American beech produced a total estimated 268,040 nuts within the 56, 0.1 ha study plots at the Savannah River Site during the 9-year study period (Table 4). Average annual

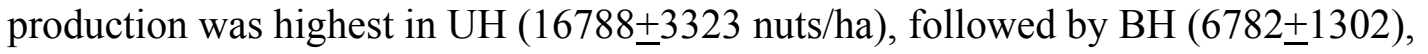
LOB (5386 \pm 1043$)$, and least in LL (1468+425), and CCLL (426 \pm 251 nuts/ha). Hard mast production was overwhelmingly dominated by Quercus nigra, which produced $62 \%$ 
of total hard mast during the study period; Q. falcata also produced $10 \%$ of total hard mast (Table 4; Fig. 5).

Nut production by all species except Fagus grandifolia, Q. incana, and Q. michauxii (which were poorly represented) differed among the 5 habitat types (Table 4). Both hickory species (Carya pallida and C. tomentosa) and two oak species (Q. coccinea and Q. velutina) produced nuts only in UH; several other oaks (Q. falcata, Q. margaretta, Q. nigra and Q. stellata) produced significantly more acorns in UH than in the other habitat types. Fagus grandifolia and Q. michauxii produced nuts in BH exclusively; Q. laurifolia and Q. nigra also were important acorn producers in BH. Quercus alba produced similar numbers of acorns in both $\mathrm{UH}$ and BH. Acorn production in LOB was dominated by Q. nigra. Quercus laevis produced acorns only in LL (Table 4). Total hard mast production varied among years, as did production by most nut-producing species (Figs. 4, 5).

\section{MANAGEMENT IMPLICATIONS}

Fruit availability at the Savannah River Site differs temporally and spatially due to differences in species composition, fruiting phenology, and the dynamic process of pioneer species' colonization and decline in recently harvested stands. Hard mast production differs temporally and spatially due to differences in the relative abundance of oaks and hickories, as well different temporal patterns of nut production among species. Land managers could enhance fruit availability for many game and nongame species by creating or maintaining young stands within forests, and by retaining a variety of habitat types with their associated fruit-producing plant species. Hard mast production could be 
enhanced by retaining important upland and bottomland hardwood forest types, and by retaining more oaks in hickories in managed stands such as pine plantation.

\section{ACKNOWLEDGMENTS}

We thank John I. Blake and the U.S. Forest Service for long term logistical and financial support of this study (No. SU96-43-F; D. J. L. and C. H. G., PIs). Support was also provided by the Department of Energy-Savannah River Operations Office through the U.S. Forest Service Savannah River under Interagency Agreement DE-IA09-76SR00056. S. Sargent, J. McCarty, S. Pearson, and C. Kwit provided on-sight leadership of the project and will be authors on resulting publications. K. Borgmann, A. Brinton, R. Busser, C. Deppe, T. Hargrave-Roof, N. Khalil, M. Reiskind, C. Renk, and E. Uramkin assisted with all surveys. 


\section{LITERATURE CITED}

BLAKE, J.G., AND W.G. HoPPES. 1986. Resource abundance and microhabitat use by birds in an isolated east-central Illinois woodlot. Auk 103:328-340.

Borgmann, K.L., S.F. Pearson, D.J. Levey, and C.H. GreenberG. 2004. Wintering yellow-rumped warblers (Dendroica coronata) track manipulated abundance of Myrica cerifera fruits. Auk 121:74-87.

CAMPO, J.J., AND G.A. HuRST. 1980. Soft mast production in young loblolly plantations. Proceedings of the Conference of Southeastern Association of Fish and Wildlife Agencies 34:470-475.

EILER, J.H., W.G. WATHEN, AND M.R. PELton. 1989. Reproduction in black bears in the southern Appalachian mountains. Journal of Wildlife Management 53:353-360.

Greenberg, C.H. And B.R. PARRESOL. 2002. Dynamics of acorn production by five species of southern Appalachian oaks. Pages 149-172 In: W.J. McShea and W.M. Healy (eds.). Oak Forest Ecosystems: Ecology and Management for Wildlife. Johns Hopkins University Press. 432pp.

GreEnBERG, C.H., AND T.G. ForReSt. 2003. Seasonal abundance of ground-occurring macroarthropods in forest and canopy gaps in the southern Appalachians. Southeastern Naturalist 2:591-608.

GreenberG, C.H., D.J. LeVEY, AND D.L. Loftis. 2007. Fruit production in mature and recently regenerated upland and cove hardwood forests of the southern Appalachians. J. Wildl. Manage. 71(2):321-329. 
HALLS, L.K., AND R. AlCANIZ. 1968. Browse plants yield best in forest openings. Journal of Wildlife Management 32:185-186.

JOHNSON, A.S., AND J.L. LANDERS. 1978. Fruit production in slash pine plantations in Georgia. Journal of Wildlife Management 42:606-613.

KoEnIG, W.D., AND R.L. MuMmE. 1987. Population ecology of the cooperatively breeding Acorn Woodpecker. Monogr. Pop. Biol. 24, Prineceton University Press.

Koenig, W.D., Mumme, R.L., Carmen, W.J., And Stanback, M.T. 1994. Acorn production by oaks in central coastal California: variation within and among years. Ecol. 75:99-109.

Kwit, C., D.J. Levey, C.H. Greenberg, S.F. Pearson, J.P. McCarty, S. SARGent, And R.L. MummE. 2004. Fruit abundance and local distribution of wintering hermit thrushes (Catharus guttatus) and yellow-rumped warblers (Dendroica coronata) in South Carolina. Auk 121(1):46-57.

LEVEY, D. J. 1990. Habitat-dependent fruiting behavior of an understory tree, Miconia centrodesma, and treefall gaps as keystone habitats for frugivores. Journal of Tropical Ecology 6:409-420.

Martin, A.C., H.S. Zim, AND A.L. Nelson. 1951. American Wildlife and Plants: A Guide to Wildlife Food Habits. Dover Publications, Inc., New York.

McCarty, J.P., D.J. Levey, C.H. Greenberg, And S. SArgent. 2002. Spatial and temporal variation in fruit use by wildlife in a forested landscape. Forest Ecology and Management 164:277-291. 
MitChell, M.S., AND R.A. PowelL. 2003. Response of black bears to forest management in the southern Appalachian mountains. Journal of Wildlife Management 67:692-705.

PERRY, R.W., R.E. ThILl, D.G. PEITZ, AND P.A. TAPPE. 1999. Effects of different silvicultural systems on initial soft mast production. Wildlife Society Bulletin 27:915-923.

Place, A.R., AND E.W. Stiles. 1991. Living off the wax of the land: bayberries and yellow-rumped warblers. Auk 109:334-345.

ROGERS, L.L. 1976. Effects of mast and berry crop failures on survival, growth and reproductive success of black bears. Transactions of the North American Wildlife and Natural Resources Conferences 41:431-438.

SKEATE, S.T. 1987. Interactions between birds and fruits in a northern Florida hammock community. Ecology 68:297-309.

StRANSKY, J.J., AND L.K. HALLS. 1980. Fruiting of woody plants affected by site preparation and prior land use. Journal of Wildlife Management 44:258-263.

StRANSKY, J.J., AND J.H. RoeSE. 1984. Promoting soft mast for wildlife in intensively managed forests. Wildlife Society Bulletin 12:234-240.

THOMPSON, J.N., AND M.F. WILLSON. 1978. Disturbance and dispersal of fleshy fruits. Science 200:1161-1163.

VAN DeRsaL, W.R. 1940. Utilization of oaks by birds and mammals. J. Wildl. Manage. $4: 404-428$.

White, D. L., AND K. F. GAINES. 2000. The Savannah River Site: site description, land use and management history. Studies in Avian Biology 21: 8-17. 
WhiteHEAD, M.A. 2003. Seasonal Variation in Food Resource Availability and Avian Communities in Four Habitat Types in the Southern Appalachian Mountains. Ph.D. Dissertation, Clemson University. 153pp.

WILLSON, M.F. 1986. Avian frugivory and seed dispersal in eastern North America. Current Ornithology 3: 223-279.

WOLFF, J.O. 1996. Population fluctuations of mast-eating rodents are correlated with production of acorns. J. Mammal. 77:850-856.

WorkMAN, S.W., AND K.W. MCLEOD. 1990. Vegetation of the Savannah River Site: Major Community Types. Savannah River Site, Aiken. 
Table 1. Proportion of total (all years and plots summed) dry edible pulp/number of fruits produced, and mean ( + SE) annual (1995-2003) soft mast production (g dry pulp/ha) by plant species and total in 5 habitat types at the Savanna River Site in South Carolina. Habitat types are upland hardwood forest, bottomland hardwood forest (BH), loblolly pine plantation (LOB), longleaf pine plantation (LL), and young (harvested in 1993) clearcuts planted in longleaf pine (CCLL). Different letters within rows denote significant differences among the habitat types. Actual means are shown here, but data were log transformed for ANOVA.

\section{SPECIES}

HABITAT TYPE

UH BH

percent

g / no.

$(n=10)$

$(\mathbf{n}=10)$

$(n=13)$

$0.0+0.0$

$0.02 / 0.03$

$0.07 / 0.15$

Arisaema triphyllum

Berchemia scandens

Calicarpa americana

Celtis occidentalis

Chionanthus virginicus

Cornus florida

Crataegus flava

Crataegus uniflora

Euonymus americanus

Galium hispidulum

Gaylussacia dumosa

G. frondosa

Ilex coriacea

I. decidua

I. glabra

I. opaca

I. verticillata

Ligustrum sinense

Lindera benzoin

Lonicera japonicum

\section{$0.46 / 3.89$}

$0.16 / 0.08$

$0.01 / 0.01$

$8.77 / 5.92$

$2.99 / 0.80$

$0.20 / 0.06$

$0.01 / 0.05$

$0.01 / 0.10$

$0.19 / 0.12$

$0.01 / 0.00$

$0.19 / 0.25$

$0.01 / 0.03$

$0.04 / 0.17$

$7.35 / 5.71$

$0.00 / 0.00$

$0.01 / 0.08$

$0.01 / 0.01$

$0.04 / 0.05$

$0.0+0.0$

$0.0 \pm 0.0^{\mathrm{A}}$

$0.0+0.0$

$43 . \overline{2}+14.0$

$3.5+1.6$

$1.9 \pm 1.4$

$200 \overline{4} .5 \pm 397.4^{\mathrm{A}}$

$55.8+22.7$

$10.6 \pm 2.5$

$1.6+1.0$

$0.0 \pm 0.0$

$0.0 \pm 0.0$

$0.0 \pm 0.0$

$0.0 \pm 0.0^{\mathrm{A}}$

$0.0+0.0$

$0.0 \pm 0.0^{\mathrm{A}}$

$518.6+154.5^{\mathrm{A}}$

$0.0 \pm 0.0$

$0.0 \pm 0.0$

$0.0+0.0$

$4.3 \pm 1.9$
$4.6+2.0^{\mathrm{B}}$

$22.4+17.3$

$23.9+8.7$

$0.0+\overline{0} .0$

$0.0 \pm 0.0$

$113.4 \pm 37.1^{\mathrm{B}}$

$0.0 \pm 0.0$

$0.0 \pm 0.0$

$0.0 \pm 0.0$

$0.0 \pm 0.0$

$0.0 \pm 0.0$

$2.6 \pm 1.7$

$57 . \overline{3}+17.9^{\mathrm{B}}$

$4.4+\overline{2} .3$

$12.5 \pm 3.9^{\mathrm{B}}$

$1706.0+427.3^{\mathrm{B}}$

$0.1+0.1$

$0.0+0.0$

$2.4+1.8$

$0.9 \pm 0.5$

$0.6+0.5$

$0.6+0.5$
$0.0+0.0^{\mathrm{A}}$

$0.0+0.0$

$11 . \overline{6}+3.9$

$1.0+\overline{0} .7$

$1.1 \pm 1.1$

$5.5 \pm 1 . \overline{4}$

$0.0+0.0$

$0.0 \pm 0.0$

$0.0 \pm 0.0$

$0.0 \pm 0.0$

$0.0 \pm 0.0^{\mathrm{A}}$

$0.0 \pm 0.0$

$0.0 \pm 0.0^{\mathrm{A}}$

$0.0+0.0^{\mathrm{C}}$

$0.0 \pm 0.0$

$0.0+0.0$

$0.0+0.0$

$0.5 \pm 0.3$

$321.6+113.9^{\mathrm{B}}$

$299.01+127.9$

$\begin{array}{ll}\mathbf{L L} & \mathbf{C C L L} \\ \mathbf{n}=\mathbf{1 3}) & \mathbf{( n = 1 0 )} \\ & \\ 0.0 \pm 0.0 & 1.5 \pm 0.7 \\ 0.0 \pm 0.0^{\mathrm{A}} & 0.0 \pm 0.0^{\mathrm{A}} \\ 0.0 \pm 0.0 & 0.0 \pm 0.0 \\ 0.0 \pm 0.0 & 58.3 \pm 31.5 \\ 32.7 \pm 12.2 & 0.0 \pm 0.0 \\ 0.0 \pm 0.0 & 0.0 \pm 0.0 \\ 90.8 \pm 44.0 \mathrm{C} & 0.0 \pm 0.0^{\mathrm{D}} \\ 135.7 \pm 105.9 & 284.6 \pm 79.4 \\ 31.7 \pm 12.2 & 2.3 \pm 0.9 \\ 0.0 \pm 0.0 & 0.0 \pm 0.0 \\ 0.0 \pm 0.0 & 2.4 \pm 1.6 \\ 44.0 \pm 33.3 & 0.3 \pm 0.3 \\ 0.0 \pm 0.0 & 0.0 \pm 0.0 \\ 0.0 \pm 0.0^{\mathrm{A}} & 0.0 \pm 0.0^{\mathrm{A}} \\ 0.0 \pm 0.0 & 0.0 \pm 0.0 \\ 0.0 \pm 0.0^{\mathrm{A}} & 0.0 \pm 0.0^{\mathrm{A}} \\ 0.2 \pm 0.2^{\mathrm{C}} & 0.0 \pm 0.0^{\mathrm{C}} \\ 0.0 \pm 0.0 & 0.0 \pm 0.0 \\ 0.0 \pm 0.0 & 2.8 \pm 2.8 \\ 0.0 \pm 0.0 & 0.0 \pm 0.0 \\ 0.0 \pm 0.0 & 5.5 \pm 3.0 \\ & \end{array}$

RM ANOVA

\begin{tabular}{lll}
\hline $\mathbf{P}_{\text {hab }}$ & $\mathbf{P}_{\mathrm{yr}}$ & $\mathbf{P}_{\text {habXyr }}$
\end{tabular}

$\begin{array}{lll}0.4212 & 0.2518 & 0.7821\end{array}$

$0.0484<0.00010 .0023$

$\begin{array}{lll}0.3378 & 0.2692 & 0.2596\end{array}$

$\begin{array}{llll}0.2275 & 0.0031 & 0.2207\end{array}$

$\begin{array}{lll}0.4833 & 0.0214 & 0.1359\end{array}$

$\begin{array}{llll}0.5368 & 0.1314 & 0.6659\end{array}$

$\begin{array}{llll}0.0033 & 0.0006 & 0.1815\end{array}$

$\begin{array}{llll}0.0731 & 0.2350 & <0.0001\end{array}$

$\begin{array}{llll}0.4336 & 0.0064 & 0.8019\end{array}$

$\begin{array}{lll}0.1979 & 0.0714 & 0.0116\end{array}$

$\begin{array}{llll}0.1335 & 0.0002 & 0.0114\end{array}$

$\begin{array}{llll}0.0583 & 0.8000 & 0.9035\end{array}$

$\begin{array}{llll}0.3378 & 0.2692 & 0.2516\end{array}$

$\begin{array}{lll}0.0148 & 0.0279 & 0.0010\end{array}$

$\begin{array}{lll}0.3378 & 0.2692 & 0.2516\end{array}$

$\begin{array}{llll}0.0100 & 0.2619 & 0.2384\end{array}$

$<0.0001<0.0001<0.0001$

$\begin{array}{lll}0.3378 & 0.2692 & 0.2516\end{array}$

$\begin{array}{lll}0.3378 & 0.2692 & 0.2516\end{array}$

$\begin{array}{lll}0.3378 & 0.2692 & 0.2516\end{array}$

$\begin{array}{llll}0.4894 & 0.0184 & 0.6207\end{array}$ 
HABITAT TYPE

\begin{tabular}{lll}
\hline UH & BH & LOB
\end{tabular}

$(\mathbf{n}=10)$

$(n=13)$

$13.3 \pm 6.1^{\mathrm{B}}$

$13.3 \pm 6.1$
$0.0 \pm 0.0$

Magnolia virginiana
Malus angustifolia

Mitchella repens

Myrica cerifera

Nyssa biflora

$N$. sylvatica

Opuntia compressa

Parthenocissus quinquefolia 0.01

Passiflora incarnata

Persea borbonia

Phytolacca americana

Prunus angustifolia

P. serotina

$P$. umbellata

Rhus copallina

$R$. radicans

$R$. toxicodendron

Rosa carolina

Rubus cuneifolius

$R$. flagellaris

Sassafras albidum

Smilax bona-nox

S. glabra

S. laurifolia

S. pumila

S. rotundifolia

S. smallii

Solanum carolinense
$0.04 / 0.06$

$0.00 / 0.01$

$0.32 / 0.98$

$0.97 / 18.72$

$4.14 / 2.88$

$0.00 / 0.00$

$2.18 / 0.10$

$0.02 / 0.02$

$0.04 / 0.05$

$0.12 / 0.15$

$1.18 / 0.15$

$2.41 / 1.45$

$0.33 / 0.12$

$2.15 / 24.90$

$0.30 / 1.21$

$1.69 / 6.87$

$<0.00 /<0.00$

$4.22 / 2.48$

$0.09 / 0.05$

$0.15 / 0.12$

$0.04 / 0.09$

$0.12 / 0.11$

$0.90 / 0.52$

$0.00 / 0.01$

$0.24 / 0.18$

$0.12 / 0.19$

$0.01 / 0.00$ $(n=10)$

$0.0 \pm 0.0^{\mathrm{A}}$

$0.3 \pm 0.1$

$37.6+16.0^{\mathrm{A}}$

$0.0 \pm 0.0^{\mathrm{A}}$

$0.0 \pm 0.0^{\mathrm{A}}$

$1.0+0.9$

$0.0+0.0^{\mathrm{A}}$

$0.0+0.0$

$0.0 \pm 0.0$

$0.9 \pm 0.4^{\mathrm{A}}$

$0.0 \pm 0.0^{\mathrm{A}}$

$0.0 \pm 0.0$

$159.8+61.4^{\mathrm{A}}$

$31.1 \pm 14.5$

$33.0+22.9^{\mathrm{A}}$

$3.7 \pm 1.2^{\mathrm{A}}$

$6.6+3.4^{\mathrm{A}}$

$0.1 \pm 0.0$

$0.0 \pm 0.0^{\mathrm{A}}$

$0.0 \pm 0.0$

$0.0+0.0$

$8.9+3.2$

$16.2+16.2$

$41.1+29.1^{\mathrm{A}}$

$1.2+0.5$

$0.0+0.0^{\mathrm{A}}$

$18.3+12.4$

$0.0 \pm 0.0^{\mathrm{A}}$
$0.0+0.0^{\mathrm{A}}$

$1252.8+301.6^{\mathrm{B}}$

$0.0+0.0$

$0.0+0.0^{\mathrm{A}}$

$4.3+4.2$

$0.0+0.0$

$12 . \overline{2}+3.1^{\mathrm{B}}$

$0.0 \pm 0.0^{\mathrm{A}}$

$0.0 \pm 0.0$

$2.8 \pm 2.5^{\mathrm{B}}$

$0.0 \pm 0.0$

$0.0+0.0^{\mathrm{B}}$

$86 . \overline{3} \pm 18.9^{\mathrm{B}}$

$0.0+0.0^{\mathrm{A}}$

$0.0 \pm 0.0$

$0.0 \pm 0.0^{\mathrm{A}}$

$0.0+0.0$

$0.0+0.0$

$0.0+0.0$

$17 . \overline{2}+2.7$

$230.8+60.6^{\mathrm{B}}$

$0.0+0.0$

$72.3 \pm 20.6^{\mathrm{B}}$

$17.6+17.3$

$0.0 \pm 0.0^{\mathrm{A}}$

$59.6+14.7^{\mathrm{B}}$

$0.0+0.0^{\mathrm{A}}$

$0.0 \pm 0.0$

$0.0 \pm 0.0^{\mathrm{C}}$

$27.9 \pm 8$.

$0.0 \pm 0.0^{\mathrm{A}}$

$0.0+0.0$

$0.7 \pm 0.4^{\mathrm{A}}$

$0.0+0.0$

$0.0 \pm 0.0$

$0.0+0.0^{\mathrm{A}}$

$0.0 \pm 0.0^{\mathrm{A}}$

$4.3+2.8$

$80.2+32.2^{\mathrm{A}}$

$0.6 \pm 0.4$

$7.0 \pm 3.0^{\mathrm{A}}$

$0.0 \pm 0.0^{\mathrm{C}}$

$149.8+40.1^{\mathrm{B}}$

$0.0 \pm 0.0$

$0.0 \pm 0.0^{\mathrm{A}}$

$0.1+0.1$

$27.7 \pm 18.3$

$0.0+0.0$

$2.7 \pm 1.2$

$0.0 \pm 0.0^{\mathrm{A}}$

$0.0+0.0$

$0.0+0.0^{\mathrm{A}}$

$0.0+0.0$

$0.0 \pm 0.0^{\mathrm{A}}$
LL

$(n=13)$

$0.0+0.0^{\mathrm{A}}$

$0.0 \pm 0.0$

$0.0 \pm 0.0^{\mathrm{C}}$

$20.1+12.2^{\mathrm{C}}$

$0.0 \pm 0.0^{\mathrm{A}}$

$0.0 \pm 0.0$

$25.4 \pm 7.0^{\mathrm{B}}$

$0.0 \pm 0.0$

$0.0 \pm 0.0$

$0.0+0.0^{\mathrm{A}}$

$0.0 \pm 0.0^{\mathrm{A}}$

$0.0 \pm 0.0$

$0.6 \pm 0.4^{\mathrm{B}}$

$1.9 \pm 1.1$

$11.8+5.8^{\mathrm{A}}$

$0.0 \pm 0.0^{\mathrm{C}}$

$16 \overline{6} .0+69.1^{\mathrm{C}}$

$0.0 \pm 0.0$

$0.1 \pm 0.1^{\mathrm{A}}$

$0.0+0.0$

$0.0+0.0$

$0.1 \pm 0.1$

$0.0+0.0$

$0.0+0.0^{\mathrm{A}}$

$0.0+0.0$

$0.0+0.0^{\mathrm{A}}$

$0.0 \pm 0.0$

$0.0 \pm 0.0^{\mathrm{A}}$
RM ANOVA

$\begin{array}{lll}\mathbf{P}_{\text {hab }} & \mathbf{P}_{\text {yr }} & \mathbf{P}_{\text {habXyr }}\end{array}$

$(\mathbf{n}=10)$

$0.0 \pm 0.0$

$0.0 \pm 0.0^{\mathrm{A}}$

$229.7 \pm 44.0^{\mathrm{D}}$

$0.0 \pm 0.0^{\mathrm{A}}$

$0.0+0.0$

$625.2+147.8^{\mathrm{C}}$

$0.0+0.0$

$5.1+5.1$

$0.0+0.0^{\mathrm{A}}$

$37.4+24.2^{\mathrm{B}}$

$350.2 \pm 256.6$

$462.9+174.1^{\mathrm{C}}$

$64.6 \pm 25.8$

$593.6+147.8^{\mathrm{C}}$

$0.0 \pm 0.0 \mathrm{C}$

$94 . \overline{2}+29.8^{\mathrm{BC}}$

$0.0 \pm 0.0$

$127 \overline{7} .9+561.8^{\mathrm{B}}$

$26.4+17.4$

$10.4 \pm 10.4$

$0.6+0.5$

$8.9+5.2$

$0.0+0.0^{\mathrm{A}}$

$0.0 \pm 0.0$

$0.0 \pm 0.0^{\mathrm{A}}$

$0.0+0.0$

$4.1 \pm 3.1^{\mathrm{B}}$

$<0.0001<0.0001<0.0001$

$\begin{array}{lll}0.1630 & 0.1266 & 0.0479\end{array}$

$<0.00010 .0006 \quad 0.0016$

$\begin{array}{llll}0.0045 & 0.0028 & 0.0105\end{array}$

$<0.0001<0.0001<0.0001$

$\begin{array}{lll}0.3378 & 0.2692 & 0.2516\end{array}$

$<0.0001<0.0001<0.0001$

$\begin{array}{lll}0.3378 & 0.2692 & 0.2516\end{array}$

$\begin{array}{llll}0.3378 & 0.2692 & 0.2516\end{array}$

$<0.0001<0.0001<0.0001$

$<0.0001<0.0001<0.0001$

$\begin{array}{lll}0.2527 & 0.0512 & 0.1903\end{array}$

$\begin{array}{lll}0.0195 & 0.0001 & 0.1532\end{array}$

$0.0972<0.00010 .0131$

$<0.0001<0.0001<0.0001$

$\begin{array}{lll}<.0001 & 0.3357 & 0.9891\end{array}$

$\begin{array}{lll}0.0232 & 0.5610 & 0.0062\end{array}$

$\begin{array}{llll}0.1941 & 0.0974 & 0.0254\end{array}$

$<0.0001<0.0001<0.0001$

$\begin{array}{lll}0.7539 & 0.4339 & 0.7539\end{array}$

$\begin{array}{llll}0.5736 & 0.2284 & 0.7301\end{array}$

$\begin{array}{lll}0.1409 & 0.0776 & 0.0313\end{array}$

$\begin{array}{lll}0.4212 & 0.1295 & 0.0138\end{array}$

$<0.0001<0.0001<0.0001$

$\begin{array}{lll}0.3378 & 0.2692 & 0.2516\end{array}$

$\begin{array}{llll}0.0062 & 0.0398 & 0.0025\end{array}$

$\begin{array}{llll}0.4490 & 0.0447 & 0.4543\end{array}$

$\begin{array}{llll}0.0791 & 0.0467 & 0.0039\end{array}$ 


\section{HABITAT TYPE}

\begin{tabular}{lll}
\hline UH & BH & LOB
\end{tabular}

\section{percent}

g / no.

$(\mathbf{n}=10)$

$(\mathbf{n}=10)$

$(n=13)$

$0.03 / 0.02$

$0.01 / 0.02$

Sorbus arbutifolia

Symplocus tinctoria

Vaccinium arboreum

$1.41 / 1.39$

V. corymbosum

V. elliottii

$V$. stamineum

Viburnum nudum

$V$. prunifolium

V. rufidulum

Vitis aestivalis

$V$. rotundifolia

$0.26 / 0.38$

$0.15 / 0.06$

$44.27 / 17.31$

$0.01 / 0.04$

$<0.00 /<0.00$

$0.03 / 0.01$

$0.55 / 0.27$

$10.93 / 1.54$

$0.0 \pm 0$

$0.0 \pm 0.0$
$0.0 \pm 0.0$

$387.6+125.5^{\mathrm{A}}$

$0.0 \pm 0.0^{\mathrm{A}}$

$0.0+0.0^{\mathrm{A}}$

$541.3+135.8^{\mathrm{A}}$

$0.0 \pm 0.0$

$0.0+0.0$

$7.4+2.9^{\mathrm{A}}$

$1.7+0.9$

$929.1 \pm 135.1^{\mathrm{A}}$

$4870.9+502.5^{\mathrm{A}}$

\section{$10.1 \pm 8.9$}

$2.3+1.2$

$78 . \overline{5}+20.4^{\mathrm{B}}$

$44.7+16.8^{\mathrm{B}}$

$4.8+2.5^{\mathrm{B}}$

$4.3 \pm 1.8$

$0.0 \pm 0.0$

$0.0 \pm 0.0^{\mathrm{B}}$

$0.0+0.0$

$135.4+33.9^{\mathrm{B}}$

$0.0+0.0$

$0.0 \pm 0.0$

$1.8 \pm 0.8^{\mathrm{B}}$

$0.0 \pm 0.0^{\mathrm{A}}$

$0.0+0.0^{\mathrm{A}}$

$1530.5+355.0^{\mathrm{C}}$

$0.0 \pm 0.0$

$0.0+0.0$

$0.2+0.2^{\mathrm{B}}$

$12.8+3.8$

$733.1 \pm 164.5^{\mathrm{C}}$
LL

$(n=13)$

$0.0+0.0$

$0.0 \pm 0.0$

$12 . \overline{2}+6.5^{\mathrm{B}}$

$0.0 \pm 0.0^{\mathrm{A}}$

$0.0+0.0^{\mathrm{A}}$

$3073.9+850.3^{\mathrm{C}}$

$0.0+0.0$

$0.0+0.0$

$0.0+0.0^{\mathrm{B}}$

$67.8+31.1$

$294.1 \pm 81.1^{\mathrm{D}}$
RM ANOVA

$\begin{array}{lll}\mathbf{P}_{\text {hab }} & \mathbf{P}_{\mathrm{yr}} & \mathbf{P}_{\text {habXyr }}\end{array}$

$(\mathbf{n}=\mathbf{1 0})$

$0.0+0.0$

$0.0 \pm 0.0$

$\begin{array}{lll}0.3378 & 0.2692 & 0.2516\end{array}$

$\begin{array}{lll}0.3378 & 0.2692 \quad 0.2516\end{array}$

$<0.0001<0.00010 .2062$

$14.8+6.3$

$<0.00010 .0116<0.0001$

$\begin{array}{lll}0.0021 & 0.0133 & 0.000\end{array}$

$0.0+0.0^{\mathrm{A}}$

$\begin{array}{llll}6862.0 \pm 2280.3^{\mathrm{D}} & 0.0003 & <0.0001 & <0.0001 \\ 0.0+0.0 & 0.3378 & 0.2692 & 0.2516\end{array}$

$0.0+0.0 \quad \mathrm{NA} \quad \mathrm{NA} \quad \mathrm{NA}$

$\begin{array}{llll}0.0+0.0^{\mathrm{B}} & 0.0007 & 0.0321 & 0.0002\end{array}$

$61.1+49.8$

$\begin{array}{lll}0.5336 & 0.0312 & 0.6122\end{array}$

$905.9 \pm 216.2^{\mathrm{C}} \quad 0.0017<0.00010 .0623$

TOTAL 
Table 2. Ripening patterns ( $g=$ green; $r=$ ripe; $d=$ damaged) of fleshy fruit-producing plant species in 5 habitat types at the Savannah River Site, SC. The period of peak abundance is in bold.

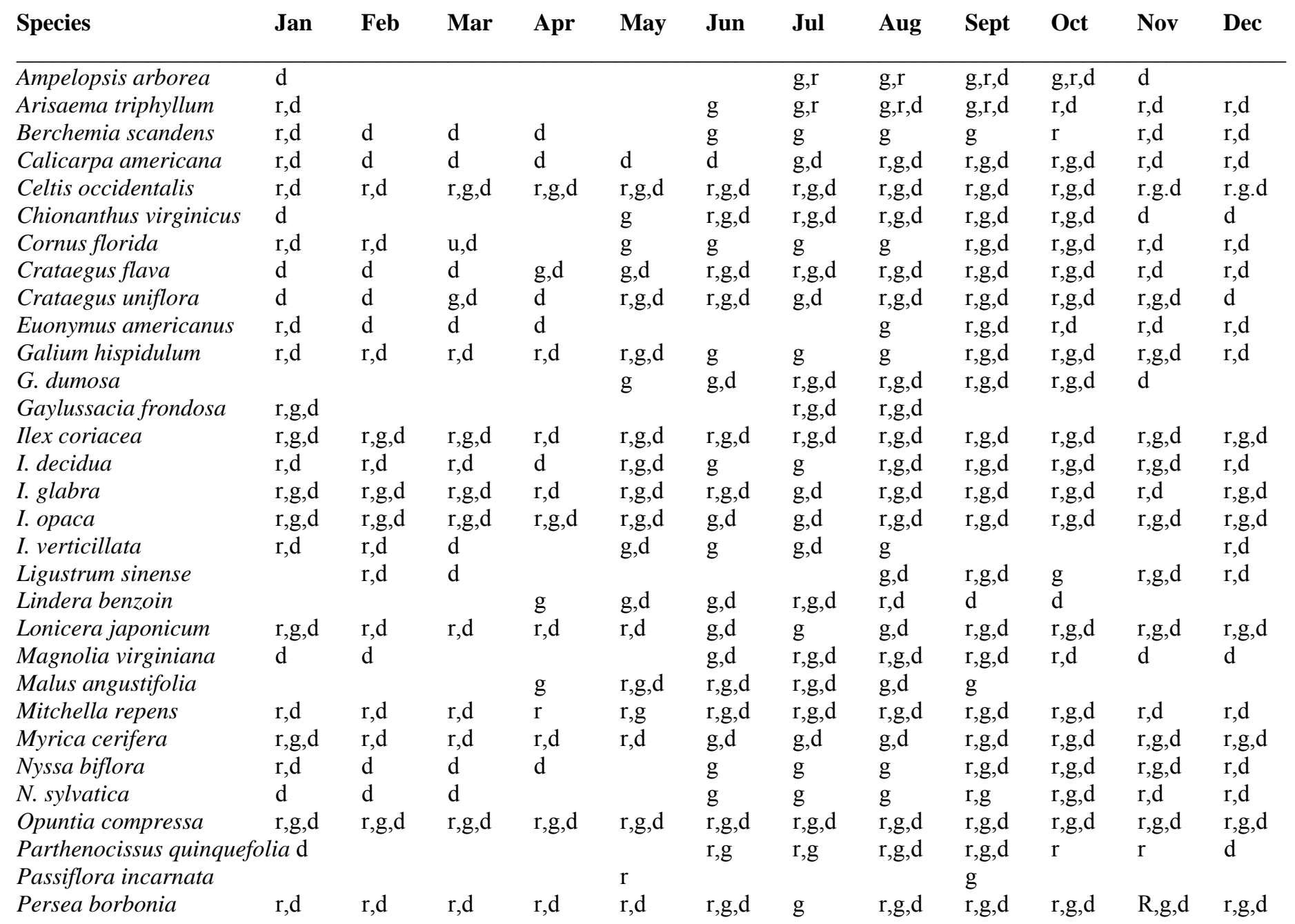




\begin{tabular}{|c|c|c|c|c|c|c|c|c|c|c|c|c|}
\hline Species & Jan & Feb & Mar & Apr & May & Jun & Jul & Aug & Sept & Oct & Nov & Dec \\
\hline Phytolacca americana & $\mathrm{d}$ & $\mathrm{d}$ & $\mathrm{r}, \mathrm{d}$ & $\mathrm{d}$ & $\mathrm{g}$ & $r, g$ & $\mathrm{r}, \mathrm{g}, \mathrm{d}$ & $r, g, d$ & $\mathrm{r}, \mathrm{g}, \mathrm{d}$ & $\mathrm{r}, \mathrm{g}, \mathrm{d}$ & $\mathrm{r}, \mathrm{g}, \mathrm{d}$ & $\mathrm{d}$ \\
\hline Prunus angustifolia & & & & $\mathrm{r}, \mathrm{g}, \mathrm{d}$ & $\mathrm{r}, \mathrm{g}, \mathrm{d}$ & $\mathrm{r}, \mathrm{g}, \mathrm{d}$ & $\mathrm{r}, \mathrm{g}, \mathrm{d}$ & d & & & & \\
\hline P. serotina & $\mathrm{d}$ & $\mathrm{d}$ & $\mathrm{d}$ & $\mathrm{g}, \mathrm{d}$ & $\mathrm{g}, \mathrm{d}$ & $\mathrm{r}, \mathrm{g}, \mathrm{d}$ & $\mathrm{r}, \mathrm{g}, \mathrm{d}$ & $\mathrm{r}, \mathrm{g}, \mathrm{d}$ & $\mathrm{r}, \mathrm{g}, \mathrm{d}$ & $d$ & $\mathrm{~d}$ & $\mathrm{~d}$ \\
\hline P. umbellata & $\mathrm{d}$ & & & $\mathrm{g}, \mathrm{d}$ & $\mathrm{r}, \mathrm{g}, \mathrm{d}$ & $\mathrm{r}, \mathrm{g}, \mathrm{d}$ & $\mathrm{r}, \mathrm{g}, \mathrm{d}$ & $\mathrm{r}, \mathrm{g}, \mathrm{d}$ & $\mathrm{r}, \mathrm{d}$ & $\mathrm{d}$ & $\mathrm{d}$ & $\mathrm{d}$ \\
\hline Rhus copallina & $\mathrm{r}, \mathrm{d}$ & $\mathrm{r}, \mathrm{d}$ & $\mathrm{r}, \mathrm{d}$ & $\mathrm{r}, \mathrm{d}$ & $\mathrm{r}, \mathrm{g}, \mathrm{d}$ & $\mathrm{r}, \mathrm{g}, \mathrm{d}$ & $\mathrm{r}, \mathrm{g}, \mathrm{d}$ & $\mathrm{r}, \mathrm{g}, \mathrm{d}$ & $\mathrm{r}, \mathrm{g}, \mathrm{d}$ & $\mathrm{r}, \mathrm{g}, \mathrm{d}$ & $\mathrm{r}, \mathrm{d}$ & $\mathrm{r}, \mathrm{d}$ \\
\hline$R$. radicans & $\mathrm{r}, \mathrm{g}, \mathrm{d}$ & $\mathrm{r}, \mathrm{g}, \mathrm{d}$ & $\mathrm{r}, \mathrm{g}, \mathrm{d}$ & d & $\mathrm{g}, \mathrm{d}$ & $\mathrm{r}, \mathrm{g}, \mathrm{d}$ & $\mathrm{r}, \mathrm{g}, \mathrm{d}$ & $\mathrm{r}, \mathrm{g}, \mathrm{d}$ & $\mathrm{r}, \mathrm{g}, \mathrm{d}$ & $\mathrm{r}, \mathrm{g}, \mathrm{d}$ & $\mathrm{r}, \mathrm{g}, \mathrm{d}$ & $\mathrm{r}, \mathrm{g}, \mathrm{d}$ \\
\hline R. toxicodendron & $\mathrm{r}, \mathrm{g}, \mathrm{d}$ & $\mathrm{r}, \mathrm{g}, \mathrm{d}$ & $\mathrm{r}, \mathrm{g}, \mathrm{d}$ & $\mathrm{r}, \mathrm{g}, \mathrm{d}$ & $\mathrm{r}, \mathrm{g}, \mathrm{d}$ & $\mathrm{r}, \mathrm{g}, \mathrm{d}$ & $\mathrm{r}, \mathrm{g}, \mathrm{d}$ & $\mathrm{r}, \mathrm{g}, \mathrm{d}$ & $\mathrm{r}, \mathrm{g}, \mathrm{d}$ & $\mathrm{r}, \mathrm{g}, \mathrm{d}$ & $\mathrm{r}, \mathrm{g}, \mathrm{d}$ & $\mathrm{r}, \mathrm{g}, \mathrm{d}$ \\
\hline Rosa carolina & $\mathrm{r}, \mathrm{d}$ & $\mathrm{r}, \mathrm{d}$ & $\mathrm{r}, \mathrm{d}$ & d & d & $\mathrm{g}$ & $\mathrm{g}$ & $\mathrm{g}, \mathrm{d}$ & $\mathrm{r}, \mathrm{g}, \mathrm{d}$ & $\mathrm{r}, \mathrm{d}$ & $\mathrm{r}, \mathrm{d}$ & $\mathrm{r}, \mathrm{d}$ \\
\hline Rubus cuneifolius & & & & $\mathrm{g}$ & $\mathrm{r}, \mathrm{g}, \mathrm{d}$ & $\mathrm{r}, \mathrm{g}, \mathrm{d}$ & $\mathrm{r}, \mathrm{g}, \mathrm{d}$ & $\mathrm{r}, \mathrm{g}, \mathrm{d}$ & d & d & & \\
\hline R. flagellaris & & & & & $\mathrm{g}$ & $\mathrm{r}, \mathrm{g}, \mathrm{d}$ & $\mathrm{r}, \mathrm{g}, \mathrm{d}$ & $\mathrm{d}$ & & & & \\
\hline Sassafras albidum & $\mathrm{d}$ & & & $\mathrm{d}$ & $\mathrm{g}$ & $\mathrm{r}, \mathrm{g}, \mathrm{d}$ & $\mathrm{r}, \mathrm{g}, \mathrm{d}$ & $\mathrm{r}, \mathrm{g}, \mathrm{d}$ & $\mathrm{r}, \mathrm{g}, \mathrm{d}$ & $\mathrm{r}, \mathrm{g}, \mathrm{d}$ & $\mathrm{r}, \mathrm{d}$ & $\mathrm{d}$ \\
\hline Smilax bona-nox & $\mathrm{r}, \mathrm{g}, \mathrm{d}$ & $\mathrm{r}, \mathrm{g}, \mathrm{d}$ & $\mathrm{r}, \mathrm{g}, \mathrm{d}$ & $\mathrm{r}, \mathrm{g}, \mathrm{d}$ & $\mathrm{r}, \mathrm{g}, \mathrm{d}$ & $\mathrm{r}, \mathrm{g}, \mathrm{d}$ & $\mathrm{r}, \mathrm{g}, \mathrm{d}$ & $\mathrm{r}, \mathrm{g}, \mathrm{d}$ & $\mathrm{r}, \mathrm{g}, \mathrm{d}$ & $\mathrm{r}, \mathrm{g}, \mathrm{d}$ & $\mathrm{r}, \mathrm{g}, \mathrm{d}$ & $\mathrm{r}, \mathrm{g}, \mathrm{d}$ \\
\hline S. glabra & $\mathrm{r}, \mathrm{d}$ & $\mathrm{r}, \mathrm{g}, \mathrm{d}$ & $\mathrm{r}, \mathrm{g}, \mathrm{d}$ & $\mathrm{r}, \mathrm{g}, \mathrm{d}$ & $\mathrm{g}, \mathrm{d}$ & $\mathrm{r}, \mathrm{g}, \mathrm{d}$ & $\mathrm{r}, \mathrm{g}, \mathrm{d}$ & r,g,d & $\mathrm{r}, \mathrm{g}, \mathrm{d}$ & $\mathrm{r}, \mathrm{g}, \mathrm{d}$ & $\mathrm{r}, \mathrm{g}, \mathrm{d}$ & $\mathrm{r}, \mathrm{g}, \mathrm{d}$ \\
\hline S. laurifolia & $\mathrm{r}, \mathrm{g}, \mathrm{d}$ & $\mathrm{r}, \mathrm{g}, \mathrm{d}$ & $\mathrm{r}, \mathrm{g}, \mathrm{d}$ & $\mathrm{r}, \mathrm{g}, \mathrm{d}$ & $\mathrm{r}, \mathrm{g}, \mathrm{d}$ & $\mathrm{r}, \mathrm{g}, \mathrm{d}$ & $\mathrm{r}, \mathrm{g}, \mathrm{d}$ & $\mathrm{r}, \mathrm{g}, \mathrm{d}$ & $\mathrm{r}, \mathrm{g}, \mathrm{d}$ & $\mathrm{r}, \mathrm{g}, \mathrm{d}$ & $\mathrm{r}, \mathrm{g}, \mathrm{d}$ & $\mathrm{r}, \mathrm{g}, \mathrm{d}$ \\
\hline S. pumila & $\mathrm{r}, \mathrm{g}, \mathrm{d}$ & $\mathrm{r}, \mathrm{g}, \mathrm{d}$ & $\mathrm{r}, \mathrm{g}, \mathrm{dg}$ & $\mathrm{g}, \mathrm{d}$ & $\mathrm{g}, \mathrm{d}$ & $\mathrm{g}, \mathrm{d}$ & $\mathrm{g}, \mathrm{d}$ & $\mathrm{g}, \mathrm{d}$ & $\mathrm{r}, \mathrm{g}, \mathrm{d}$ & $\mathrm{r}, \mathrm{g}, \mathrm{d}$ & $\mathrm{r}, \mathrm{g}, \mathrm{d}$ & $\mathrm{r}, \mathrm{g}, \mathrm{d}$ \\
\hline S. rotundifolia & $\mathrm{r}, \mathrm{d}$ & $\mathrm{r}, \mathrm{d}$ & $\mathrm{r}, \mathrm{d}$ & $\mathrm{r}, \mathrm{g}, \mathrm{d}$ & $\mathrm{r}, \mathrm{g}, \mathrm{d}$ & $\mathrm{r}, \mathrm{g}, \mathrm{d}$ & $\mathrm{r}, \mathrm{g}, \mathrm{d}$ & $\mathrm{r}, \mathrm{g}, \mathrm{d}$ & $\mathrm{r}, \mathrm{g}, \mathrm{d}$ & $\mathrm{r}, \mathrm{g}, \mathrm{d}$ & $\mathrm{r}, \mathrm{g}, \mathrm{d}$ & $\mathrm{r}, \mathrm{d}$ \\
\hline S. smallii & $\mathrm{r}, \mathrm{g}, \mathrm{d}$ & $\mathrm{r}, \mathrm{g}$ & $\mathrm{r}, \mathrm{g}$ & $\mathrm{g}$ & $\mathrm{g}$ & $r, g$ & $\mathrm{r}, \mathrm{g}, \mathrm{d}$ & $\mathrm{r}, \mathrm{g}, \mathrm{d}$ & $\mathrm{r}, \mathrm{g}, \mathrm{d}$ & $\mathrm{r}, \mathrm{g}, \mathrm{d}$ & $\mathrm{r}, \mathrm{g}, \mathrm{d}$ & $\mathrm{r}, \mathrm{g}, \mathrm{d}$ \\
\hline Solanum carolinense & $\mathrm{r}, \mathrm{d}$ & $\mathrm{r}, \mathrm{d}$ & $\mathrm{r}, \mathrm{d}$ & $\mathrm{d}$ & $\mathrm{d}$ & $\mathrm{g}$ & $\mathrm{g}, \mathrm{d}$ & $\mathrm{r}, \mathrm{g}, \mathrm{d}$ & $\mathrm{r}, \mathrm{g}, \mathrm{d}$ & $\mathrm{r}, \mathrm{g}, \mathrm{d}$ & $\mathrm{r}, \mathrm{d}$ & $\mathrm{r}, \mathrm{d}$ \\
\hline Sorbus arbutifolia & d & $\mathrm{d}$ & $\mathrm{d}$ & $\mathrm{g}, \mathrm{d}$ & $\mathrm{g}, \mathrm{d}$ & $\mathrm{g}$ & $\mathrm{g}$ & $\mathrm{g}, \mathrm{d}$ & $\mathrm{r}, \mathrm{g}$ & $\mathrm{r}, \mathrm{g}, \mathrm{d}$ & $\mathrm{r}, \mathrm{d}$ & $\mathrm{r}, \mathrm{d}$ \\
\hline Symplocus tinctoria & d & & & & $\mathrm{g}$ & $\mathrm{g}$ & $\mathrm{g}$ & $\mathrm{r}, \mathrm{g}, \mathrm{d}$ & $\mathrm{R}, \mathrm{g}, \mathrm{d}$ & $\mathrm{r}, \mathrm{g}, \mathrm{d}$ & $r, g, d$ & $\mathrm{r}, \mathrm{d}$ \\
\hline Vaccinium arboreum & $\mathrm{r}, \mathrm{g}, \mathrm{d}$ & $\mathrm{r}, \mathrm{d}$ & $\mathrm{r}, \mathrm{d}$ & $\mathrm{r}, \mathrm{d}$ & $\mathrm{r}, \mathrm{g}, \mathrm{d}$ & $\mathrm{g}, \mathrm{d}$ & $\mathrm{r}, \mathrm{g}, \mathrm{d}$ & $\mathrm{r}, \mathrm{g}, \mathrm{d}$ & $r, g, d$ & $\mathrm{R}, \mathrm{g}, \mathrm{d}$ & $\mathrm{R}, \mathrm{g}, \mathrm{d}$ & $\mathrm{R}, \mathrm{g}, \mathrm{d}$ \\
\hline V. corymbosum & & & & g,d & $\mathrm{r}, \mathrm{g}, \mathrm{d}$ & $\mathrm{r}, \mathrm{g}, \mathrm{d}$ & $\mathrm{r}, \mathrm{g}, \mathrm{d}$ & $\mathrm{r}, \mathrm{g}, \mathrm{d}$ & $\mathrm{r}, \mathrm{g}, \mathrm{d}$ & & & \\
\hline V. elliottii & & & & $\mathrm{g}$ & $\mathrm{r}, \mathrm{g}, \mathrm{d}$ & $\mathrm{r}, \mathrm{g}, \mathrm{d}$ & $\mathrm{r}, \mathrm{g}, \mathrm{d}$ & $\mathrm{g}, \mathrm{d}$ & & & & \\
\hline$V$. stamineum & $\mathrm{d}$ & & g & $\mathrm{g}$ & $\mathrm{g}, \mathrm{d}$ & $\mathrm{r}, \mathrm{g}, \mathrm{d}$ & $\mathrm{r}, \mathrm{g}, \mathrm{d}$ & $\mathrm{r}, \mathrm{g}, \mathrm{d}$ & $\mathrm{r}, \mathrm{g}, \mathrm{d}$ & $\mathrm{r}, \mathrm{g}, \mathrm{d}$ & $\mathrm{r}, \mathrm{d}$ & $\mathrm{r}, \mathrm{d}$ \\
\hline Viburnum nudum & $\mathrm{r}, \mathrm{d}$ & $\mathrm{d}$ & $\mathrm{r}, \mathrm{d}$ & $\mathrm{r}, \mathrm{d}$ & d & $\mathrm{g}, \mathrm{d}$ & $\mathrm{g}, \mathrm{d}$ & $\mathrm{g}, \mathrm{d}$ & $\mathrm{r}, \mathrm{g}, \mathrm{d}$ & $\mathrm{r}, \mathrm{g}, \mathrm{d}$ & $\mathrm{r}, \mathrm{g}, \mathrm{d}$ & $\mathrm{r}, \mathrm{d}$ \\
\hline V. prunifolium & d & $\mathrm{d}$ & & & & & $\mathrm{g}$ & $\mathrm{g}$ & $\mathrm{g}$ & r & $\mathrm{d}$ & $\mathrm{d}$ \\
\hline V. rufidulum & $\mathrm{r}, \mathrm{d}$ & $\mathrm{d}$ & $\mathrm{d}$ & $\mathrm{d}$ & g,d & g,d & $\mathrm{g}, \mathrm{d}$ & $\mathrm{g}, \mathrm{d}$ & $\mathrm{r}, \mathrm{g}, \mathrm{d}$ & $\mathrm{r}, \mathrm{g}, \mathrm{d}$ & $r, g, d$ & $\mathrm{r}, \mathrm{d}$ \\
\hline Vitis aestivalis & $\mathrm{g}, \mathrm{d}$ & $\mathrm{g}, \mathrm{d}$ & $\mathrm{g}, \mathrm{d}$ & $\mathrm{d}$ & $\mathrm{g}, \mathrm{d}$ & $\mathrm{g}, \mathrm{d}$ & $\mathrm{r}, \mathrm{g}, \mathrm{d}$ & $\mathrm{r}, \mathrm{g}, \mathrm{d}$ & $\mathrm{r}, \mathrm{g}, \mathrm{d}$ & $\mathrm{r}, \mathrm{g}, \mathrm{d}$ & $\mathrm{r}, \mathrm{d}$ & $\mathrm{r}, \mathrm{d}$ \\
\hline V. rotundifolia & $\mathrm{r}, \mathrm{d}$ & d & $\mathrm{r}, \mathrm{d}$ & & $\mathrm{r}$ & $\mathrm{g}, \mathrm{d}$ & $\mathrm{r}, \mathrm{g}, \mathrm{d}$ & $\mathrm{r}, \mathrm{g}, \mathrm{d}$ & $\mathrm{r}, \mathrm{g}, \mathrm{d}$ & $\mathrm{r}, \mathrm{g}, \mathrm{d}$ & $\mathrm{r}, \mathrm{g}, \mathrm{d}$ & $\mathrm{d}$ \\
\hline
\end{tabular}


Table 3. Mean ( \pm SE) number of fruits produced per individual plant (1999-2003). Fruit yield for trees is based on fruits per individual $\left(\mathrm{m}^{3}\right.$ for $V$. stamineum; number $\geq 2.4 \mathrm{~cm} \mathrm{dbh}$ for C. florida and I. opaca; total individuals for $M$. cerifera) using only plants known to fruit during the study period, and per total number of individuals including individuals that never fruited during the study period.

Known Fruiters Total Individuals

\begin{tabular}{|c|c|c|}
\hline Species & Mean_SE & Mean_SE \\
\hline Cornus florida & $240.2+66.1$ & $192.6 \pm 53.0$ \\
\hline Ilex ораса & $97.5 \pm 48.5$ & $37.8 \pm \overline{1} 8.8$ \\
\hline Myrica cerifera & $491 . \overline{1}+97.9$ & $380 . \overline{9} \pm 75.9$ \\
\hline Vaccinium stamineum & $37.0 \pm 18.2$ & $37.0 \pm 18.2$ \\
\hline
\end{tabular}


Table 4. Proportion of total (all years and plots summed) hard mast (acorns, hickory nuts, and beech nuts) produced, and mean ( \pm SE) annual (1995-2003) hard mast production (number/ha) by species and total in 5 habitat types at the Savannah River Site in South Carolina. Habitat types are upland hardwood forest (UH), bottomland hardwood forest (BH), loblolly pine plantation (LOB), longleaf pine plantation (LL), and young (harvested in 1993) clearcuts planted in longleaf pine (CCLL). Actual means are shown here, but data were log transformed for ANOVA.

\section{HABITAT TYPE}

\section{$\overline{\mathrm{UH}}$}

$(\mathbf{n}=10)$

BH

$(\mathbf{n}=10)$

LOB

$(\mathbf{n}=13)$
SPECIES

\begin{tabular}{ll} 
& \\
& $\begin{array}{l}\text { Total } \\
\end{array}$ \\
& \\
\hline & \\
Carya pallida & 3.4 \\
C. tomentosa & 2.7 \\
Fagus grandifolia & 0.2 \\
Quercus alba & 3.4 \\
Q. coccinea & 0.6 \\
Q. falcata & 9.7 \\
Q. incana & 1.8 \\
Q. laevis & 0.8 \\
Q. laurifolia & 4.3 \\
Q. margaretta & 4.2 \\
Q. michauxii & 0.4 \\
Q. nigra & 62.2 \\
Q. stellata & 5.2 \\
Q. velutina & 1.1
\end{tabular}

$1092.2 \pm 276.0^{\mathrm{A}}$ $859.6+163.5^{\mathrm{A}}$

$0.0+0.0$

$533.0+164.2^{\mathrm{A}}$

$184.7+61.3^{\mathrm{A}}$

$2828 . \overline{7}+751.8^{\mathrm{A}}$

$0.0+0.0$

$0.0 \pm 0.0^{\mathrm{A}}$

$407.3+174.4^{\mathrm{A}}$

$668.7+668.7^{\mathrm{A}}$

$0.0 \pm 0.0 \mathrm{C}$

$4272.4 \pm 1080.3$

$\begin{array}{ll}1490.6 \pm 225.1^{\mathrm{A}} & 170.0 \pm 78.2^{\mathrm{B}} \\ 363.2+363.2^{\mathrm{A}} & 0.0 \pm 0.0^{\mathrm{B}}\end{array}$

$16788.4+3323.0^{\mathrm{A}} \quad 6782.0 \pm 1301.9^{\mathrm{B}} \quad 5385.6+1043.6^{\mathrm{C}} \quad 1468.2+424.5^{\mathrm{D}} \quad 425.8+250.6^{\mathrm{D}}$

$0.0 \pm 0.0^{\mathrm{B}}$

$0.0+0.0^{\mathrm{B}}$

$0.0 \pm 0.0$

$0.0+0.0^{\mathrm{B}}$

$0.0+0.0^{\mathrm{B}}$

$225.0+108.8^{\mathrm{C}}$

132.161 .0

$0.0+0.0^{\mathrm{A}}$

$0.0+0.0^{\mathrm{C}}$

$51.5 \pm 27.0^{\mathrm{C}}$

$0.0 \pm 0.0^{\mathrm{C}}$

$4937.7 \pm 990.2^{\mathrm{C}}$

$0.0 \pm 0.0^{\mathrm{C}}$

$0.0+0.0^{\mathrm{B}}$
TOTAL
LL

$(n=13)$

RM ANOVA

CCLL

$\overline{\mathbf{P}_{\text {hab }}} \quad \mathbf{P}_{\text {year }} \quad \mathbf{P}_{\text {yearxhab }}$

$(n=10)$

$0.0+0.0^{\mathrm{B}} \quad 0.0+0.0^{\mathrm{B}}$

$0.0 \pm 0.0 \quad 0.0+0.0$

$0.0 \pm 0.0^{\mathrm{B}} \quad 0.0 \pm 0.0^{\mathrm{B}}$

$0.0+0.0^{\mathrm{B}} \quad 0.0+0.0^{\mathrm{B}}$

$0.0 \pm 0.0^{\mathrm{B}} \quad 0.0 \pm 0.0^{\mathrm{B}}$

$308.6+136.5 \quad 0.0+0.0$

$199.7 \pm 58.9^{\mathrm{B}} \quad 0.0 \pm 0.0^{\mathrm{A}}$

$0.4 \pm 0.4^{\mathrm{C}} \quad 0.0+0.0^{\mathrm{C}}$

$291.6+151.5^{\mathrm{C}}$

$0.0 \pm 0.0 \mathrm{D}$

$300.9+228.5^{\mathrm{BC}}$

$0.0 \pm 0.0^{\mathrm{D}}$

$88.4 \pm 34.5^{\mathrm{D}}$

$0.0 \pm 0.0^{\mathrm{C}}$

$0.0+0.0^{\mathrm{B}}$

$<0.0001<0.0001<0.0001$

$<0.0001<0.0001<0.000$

$\begin{array}{lll}0.3289 & 0.2851 & 0.2376\end{array}$

$\begin{array}{llll}0.0091 & 0.0010<0.0001\end{array}$

$\begin{array}{llll}0.0053 & 0.0587 & 0.0121\end{array}$

$\begin{array}{llll}0.0008 & 0.2282 & 0.0429\end{array}$

$\begin{array}{lll}0.4690 & 0.2728 & 0.4946\end{array}$

$\begin{array}{lll}0.0346 & 0.4744 & 0.3412\end{array}$

$\begin{array}{lll}0.0126 & 0.0115 & 0.0050\end{array}$

$\begin{array}{llll}0.0457 & 0.7969 & 0.3451\end{array}$

$\begin{array}{lll}0.3454 & 0.2946 & 0.2349\end{array}$

$0.0359<0.0001<0.0001$

$\begin{array}{llll}<0.0001 & 0.0935 & 0.1426\end{array}$

$\begin{array}{lll}0.0026 & 0.0349 & 0.0011\end{array}$ 


\section{List of Figures}

1. Mean (+SE) annual total dry pulp biomass (g/ha) of soft mast in 5 habitat types at the Savanna River Site, SC 1995-2003.

2. Mean monthly total dry pulp biomass ( $\mathrm{g} / \mathrm{ha}$ ) of soft mast in 5 habitat types at the Savannah River Site, SC 1995-2003.

3. Mean monthly dry pulp biomass (g/ha) of important fruit-producing species in 5 habitat types at the Savannah River Site, SC 1995-2003.

4. Mean annual hard mast (acorns, hickory nuts and beechnuts) production (number/ha) in 5 habitat types at the Savannah River Site, SC 1995-2003.

5. Mean annual number of Q. nigra and Q. falcata acorns (number/ha) in 5 habitat types at the Savannah River Site, SC 1995-2003. 


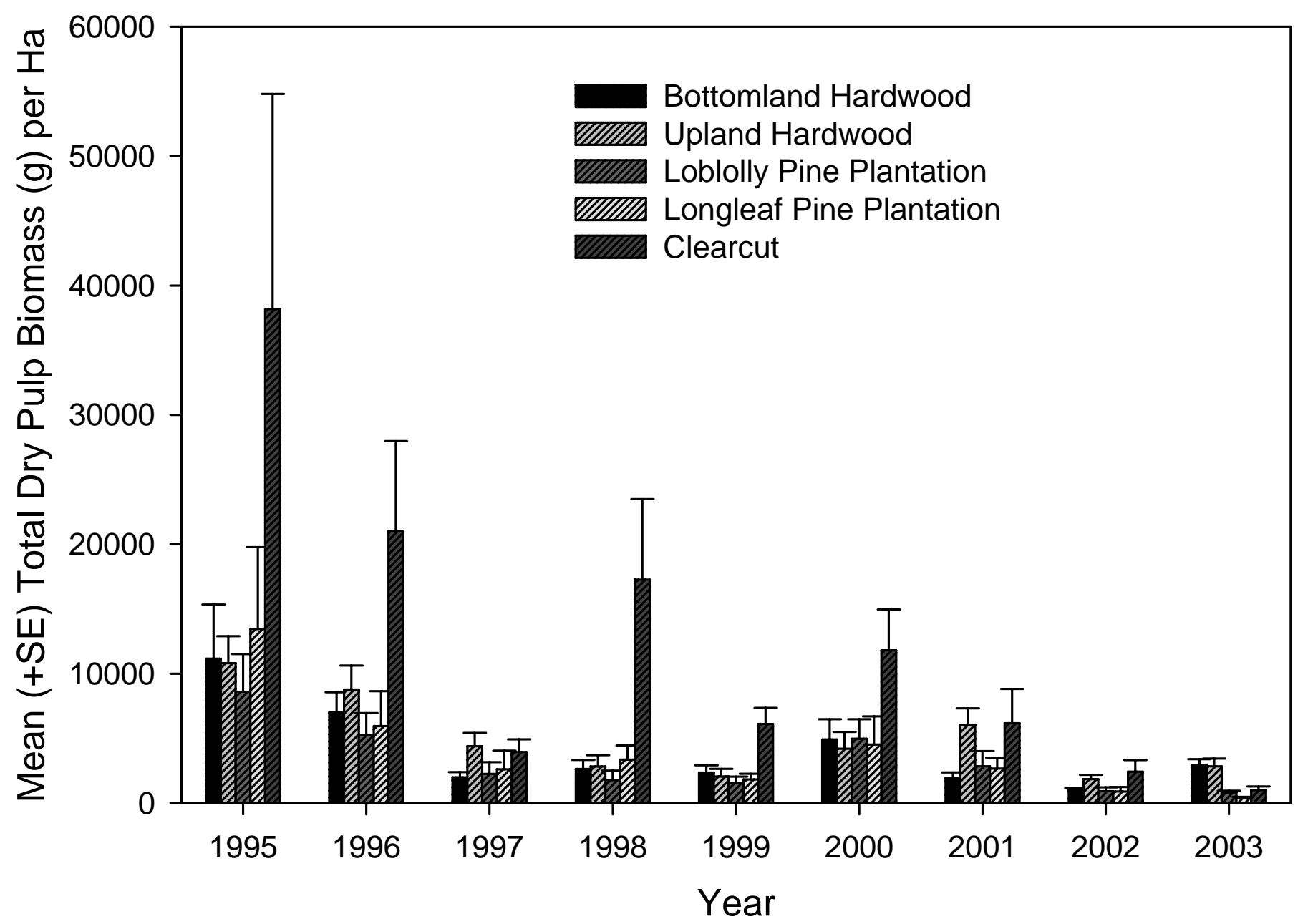




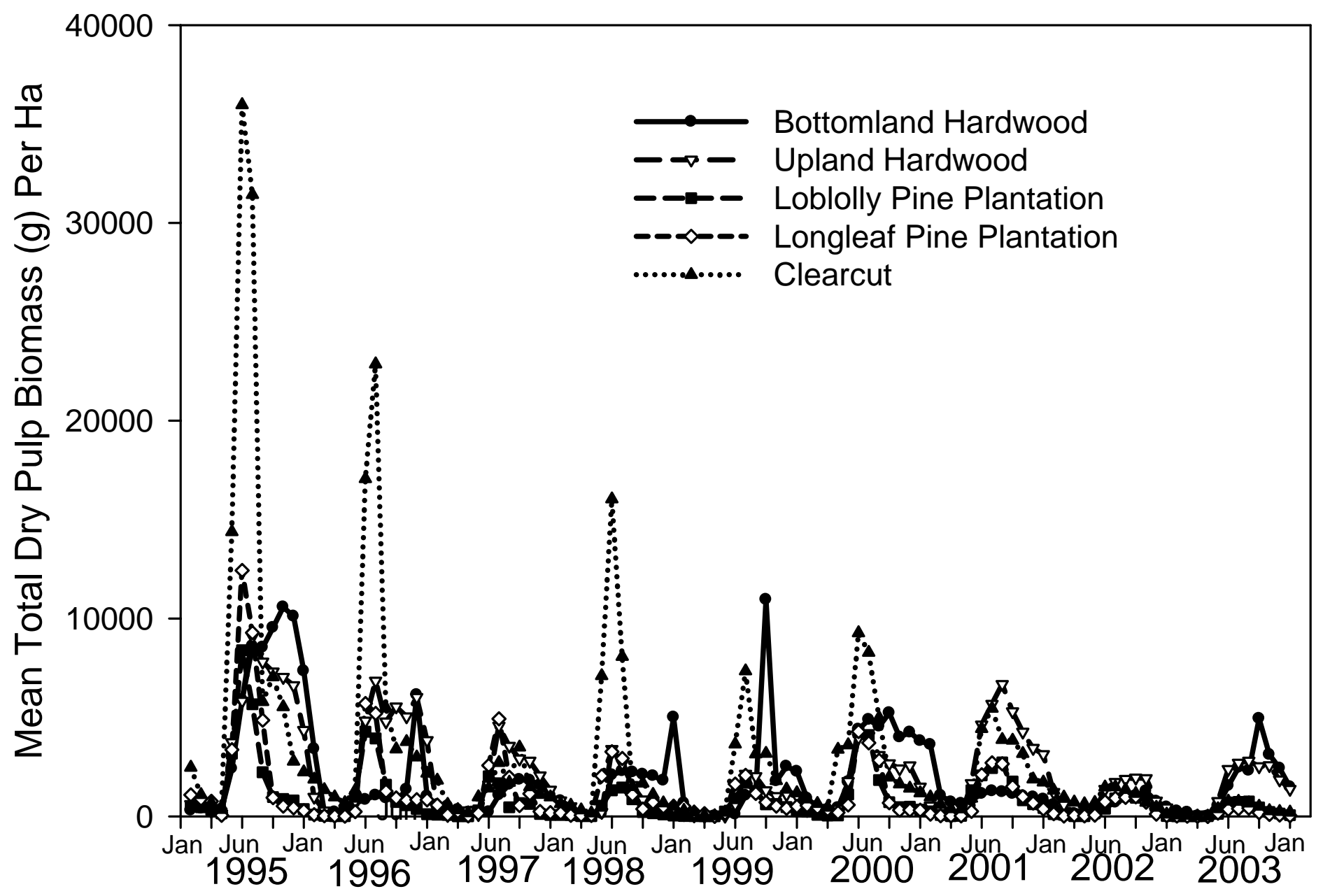




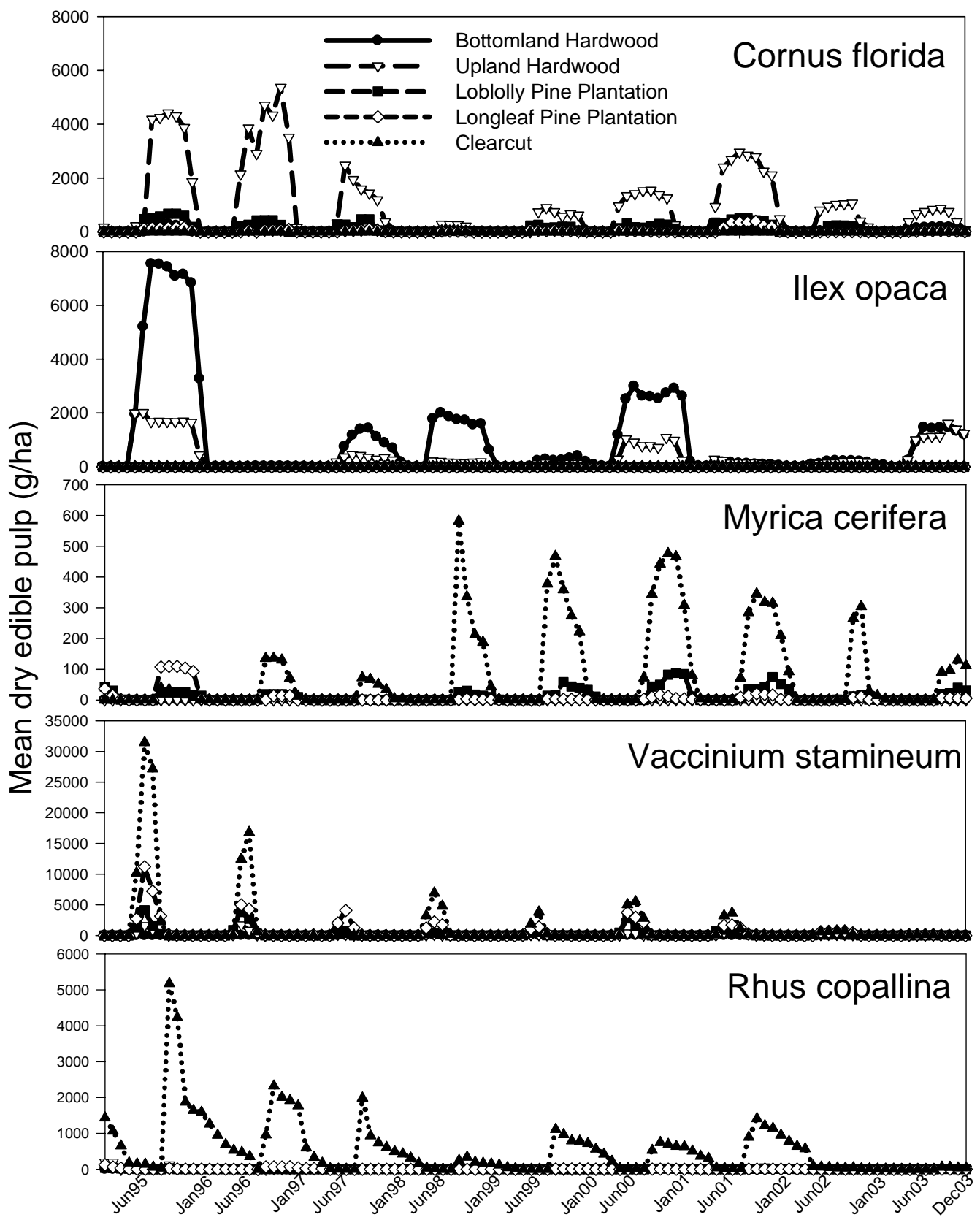




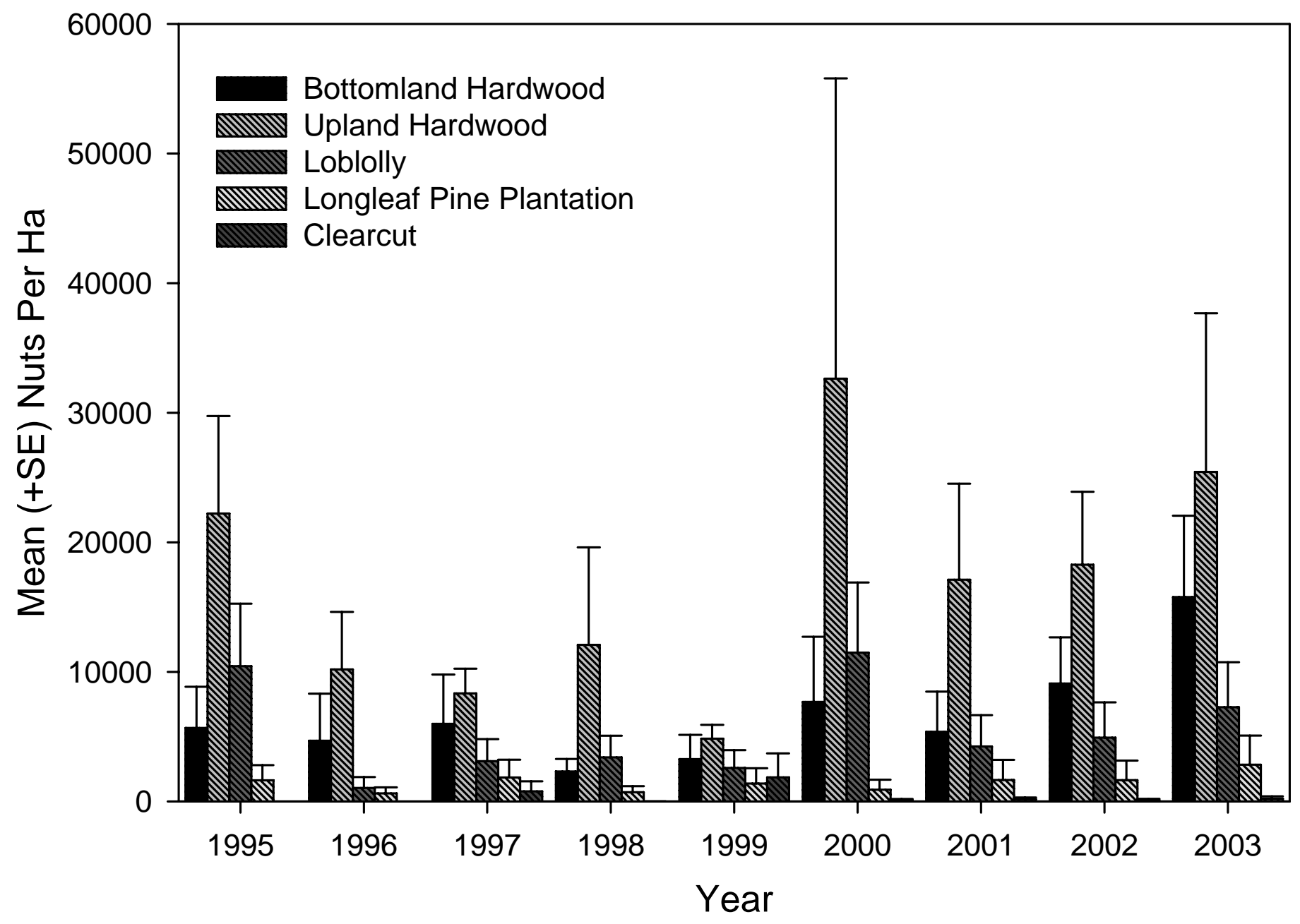




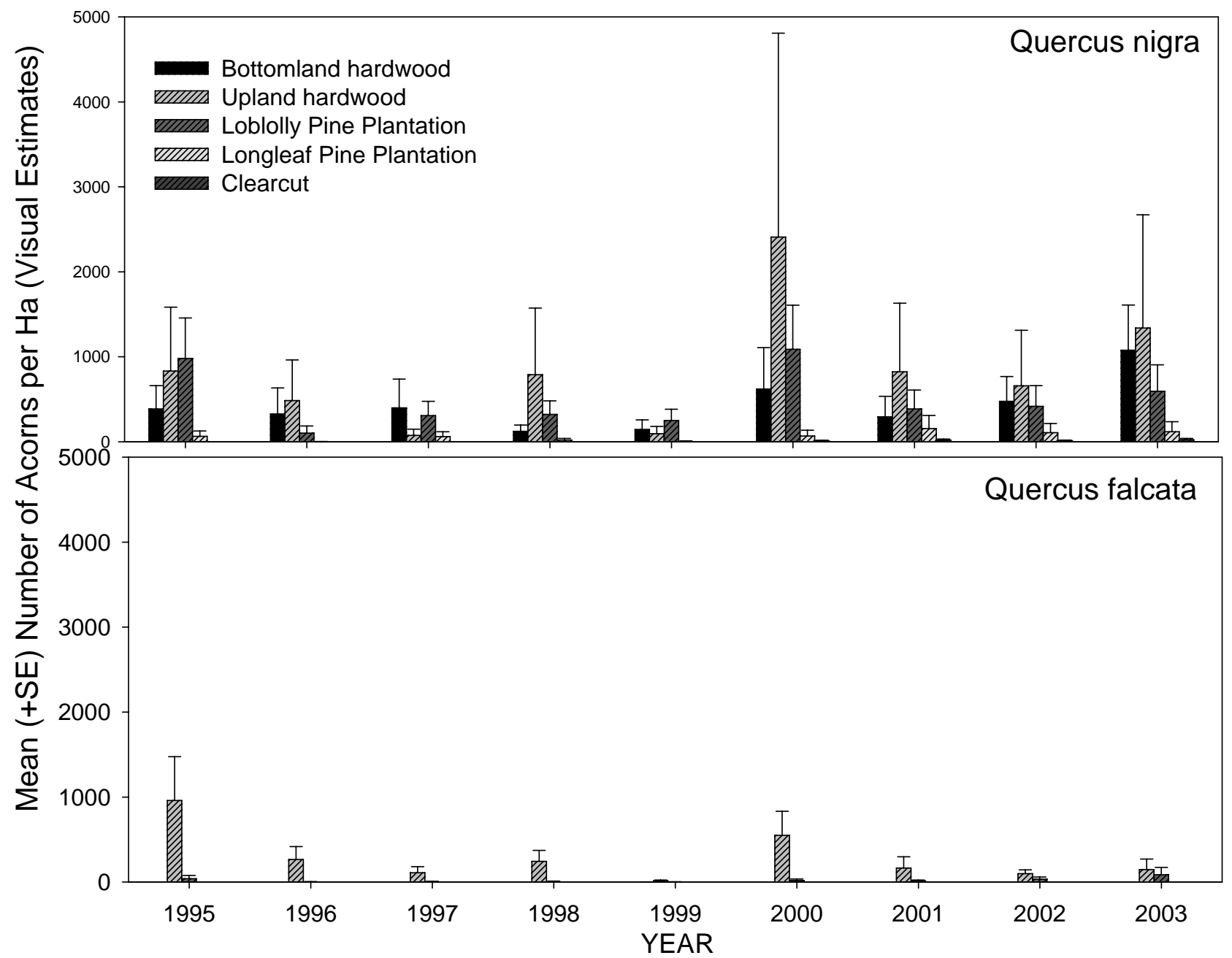




\title{
Section 2
}

\section{Comparison of fruit consumption by fledgling versus adult birds at the Savann: River Site}

\author{
Abstract: We report stable isotope ratios of nitrogen in feathers and blood of common omnivorous birds \\ at the Savannah River Station, near Aiken, SC. We predicted that ratios would be higher in adult birds \\ than in fledgling birds of the same species. We also predicted that ratios would be greater at the tip of \\ tail feathers from fledgling birds than at the base of feathers. Support of the first prediction would \\ suggest that fledgling birds rely more heavily than adult birds on fruits. Support of the second \\ prediction would suggest that young birds are especially dependent on fruits during the period when \\ their parents have stopped feeding them and they are learning to forage on their own. Support of either \\ prediction would provide rationale for management of habitat to produce fruit during mid-summer, \\ when fledgling birds are most abundant and presumably most dependent on fruit. We attained sufficient \\ sample sizes for seven species of omnivorous birds: Carolina Chickadee, Northern Cardinal, Tufted \\ Titmouse, Pine Warbler, Red-eyed Vireo, White-eyed Vireo, and Summer Tanager. Isotopic signatures \\ of nitrogen were essentially identical for adult versus fledgling birds and for the tips and bases of tail \\ feathers from fledgling birds. Thus, neither prediction was supported for any species. We conclude that \\ fruits may be equally important to adult and fledgling birds or that use of stable isotopes may not be \\ allow sufficient resolution to detect differences in diet over an ecologically relevant time scale for \\ fledgling birds.
}

\section{Introduction}


Fruit production in North America is typically viewed as important for migratory birds, which must eat large quantities of highly caloric food to fatten for migration. Because fruits are generally high in carbohydrates or fat, and are relatively easy to find, capture, and process, reliance on them during times of high energy demand is reasonable to expect - it's presumably easier to fatten on fruits than on insects, which are relatively difficult to find, capture, and process. Fruits, however, may also be important at other times of year for other types of birds. In particular, we hypothesize that fruits might provide an important energy source for young birds, immediately after they have fledged from the nest and are learning to forage on their own. During this time, parents provide gradually less food but the fledglings have not yet acquired the foraging skills needed to efficiently find and capture insects. In eastern North America, they are known to move into areas of second growth during the first month after fledgling. This behavioral shift in habitat preference provides circumstantial evidence for our hypothesis because fruits are far more abundant in second growth habitats than in the forests and edge habitats where many migratory songbirds nest.

Documenting diets of fledgling birds is extraordinarily difficult because they are secretive -- they frequent dense tangles of vegetation, stay low to the ground, don't make noise, and fly only short distances. Thus, it's nearly impossible to observe them in the field and their natural history remains poorly understood. One approach to documenting their diet is to capture them and collect fecal samples. Fecal samples, however, provide only a "snapshot" of what a given bird eats at a given time. Furthermore, because fruits and insects pass through the gut at different rates, fecal samples yield a biased estimate of food choice. An alternative approach, rapidly gaining acceptance, is the use of stable isotopes. Isotopic ratios of nitrogen ("delta-N") are especially pertinent for distinguishing consumption of fruit versus insects because nitrogen ratios become "enriched" (greater in value) at higher trophic levels. Thus, birds consuming a greater quantity of fruits would 
be expected to have lower isotopic ratios of nitrogen in their tissues than birds consuming insects or other animals that are higher on the food chain.

We used stable isotope ratios of nitrogen to test two predictions of the importance of fruit for fledgling birds: (1) Recently fledged individuals of omnivorous species will be more reliant on fruit than adult birds of the same species in the same habitat. Recently fledged individuals will thus have lower delta-N values than adults. (2) Recently fledged individuals of omnivorous species will be more reliant on fruit after they have almost completely grown their tail feathers compared to when their tail feathers are first appearing and they are presumably being fed insects by their parents. This prediction would be supported by lower delta-N ratios in the base of tail feathers than at their tip. We tested these predictions in seven species of omnivorous songbirds.

\section{Methods}

This study took place at the U.S. Department of Energy's Savannah River Site, a National Environmental Research Park located in Aiken and Barnwell Counties, South Carolina, U.S.A. (33 $\left.{ }^{\circ} 18^{\prime} \mathrm{N}, 81^{\circ} 37^{\prime} \mathrm{W}\right)$. The site lies within the Sandhill and upper Coastal Plain physiographic provinces. Forested areas cover $>80 \%$ of the site (Workman and McLeod 1990). The majority of this area consists of a patchy mosaic of managed longleaf pine (Pinus palustris) and loblolly pine (P. taeda) stands, and to a lesser extent, upland and bottomland hardwood stands.

In June and July of 2006, we used mist nets ( $3 \times 12 \mathrm{~m} ; 36 \mathrm{~mm}$ mesh) to capture birds in several areas of second growth vegetation. Most of these areas had been clearcut 2-4 years previously and had been replanted with pin seedlings. For a description of the fruits available in this habitat type see Section 1. All birds were weighed, measured, sexed (when possible), and aged according to Pyle (2002). We distinguished between After Hatch 
Year (AHY or adult) birds and Hatch Year (HY or fledgling) birds. For some species, it was possible to determine Second Year (SY) individuals but for analyses, these individuals were pooled with AHY individuals. We did not target any particular species.

For each captured bird, we plucked a single tail feather, a single pin feather (when possible), and took a blood sample from the bird's brachial vein. All samples were stored frozen. Prior to analysis, feathers were cleaned of lipids following procedures described in Pearson et al (2003). Analyses for delta-N were completed at the Savannah River Ecology Lab by Chris Romaneck.

Isotopic ratios of nitrogen were statistically analyzed using t-tests for comparisons of delta- $\mathrm{N}$ of blood and pin feathers between HY and AHY individuals of a given species (prediction 1). For comparisons of delta-N between the tip and base of tail feathers (prediction 2), we used paired-t tests.

\section{Results}

We attained sufficient sample sizes for seven species: Carolina Chickadee $(n=27)$, Tufted Titmouse $(n=42)$, Northern Cardinal $(n=63)$, Pine Warbler $(n=124)$, Red-eyed Vireo $(n=109)$, White-eyed Vireo $(n=60)$, and Summer Tanager $(n=24)$. Of these the proportion of HY individuals was: $52 \%$ for Carolina Chickadee, $45 \%$ for Tufted Titmouse, $47 \%$ for Northern Cardinal, $74 \%$ for Pine Warbler, 23\% for Red-eyed Vireo, $52 \%$ for White-eyed Vireo, and 27\% for Summer Tanager.

Average delta-N values for pin feathers ranged from 2.1 (Pine Warbler) to 4.5 (Tufted Titmouse), indicating a wide range of diets among species. With respect to Prediction \#1, however, delta-N values did not differ between adult (AHY) and fledgling (HY) individuals for any species (P’s $>0.25$; Figure 1). Likewise, delta-N 
values of blood did not differ between AHY and HY individuals for any species (P's $>0.25$; Figure 2). These results lead us to reject Prediction \#1.

Delta-N values were consistently higher at the base of tail feathers than at their tips for HY individuals, although this varied considerably among the seven focal species and was not significant for any species (P's > 0.25 ; Figure 3). Note that the direction of difference, although consistent among species is in the opposite direction than expected. Thus, we reject Prediction \#2.

\section{Discussion}

Despite much variation in delta- $\mathrm{N}$ values among species, we found no pattern of variation within species. This suggests that HY and AHY individuals eat similar diets, at least with respect to the trophic level of items included in their diets. We found no support for either prediction and conclude that HY birds are no more reliant on fruits than AHY birds. Likewise, $\mathrm{HY}$ individuals do not become increasingly reliant of fruits as they first start to forage on their own; their tail feathers show no hint of a shift in diet between the time those feathers are first start to be grown (when being fed by parents) and the time when those feathers complete growth (when fledgling primarily feed themselves).

We cannot dismiss our results on the basis of low sample sizes. Not only were sample sizes generally greater than approximately 30 , but no P-values were even close to being significant. And, for prediction \#2, the observed difference was in the opposite direction expected.

Either isotopic ratios do not provide the temporal resolution necessary to track diet shifts over the course of a fledgling bird's life (which we view as unlikely) or HY and AHY individuals do not substantially differ in their 
diets. We suspect the latter. This does not mean, however, that fruit is unimportant to fledgling birds - only that fruit is not more important to fledgling birds than to adult birds. It remains to be determined just how important fruit is to omnivorous birds in the summer, after nesting season has stopped and molt has begun. A distinct possibility is that fruit is highly important for some species, young and old, during this time of year. Documenting this through the use of stable isotopes will require application of mixing models, as described in Section 3. 
Figure 1. Delta-N values of pin feathers sampled from After Hatching Year (AHY or adult) and Hatching Year (HY or fledgling) of seven omnivorous bird species in June and July of 2006 at the Savannah River Site, Aiken, South Carolina. $\mathrm{CACH}=$ Carolina Chickadee; ETTI $=$ Tufted Titmouse; NOCA $=$ Northern Cardinal; PIWA = Pine Warbler; REVI $=$ Red-eyed Vireo; SUTA $=$ Summer Tanager; WEVI $=$ White-eyed Vireo. Bars are standard deviations. All differences between AHY and HY within a given species are not significant $(\mathrm{P}>0.25)$.

Delta-N values from Pin feathers of AHY and HY birds

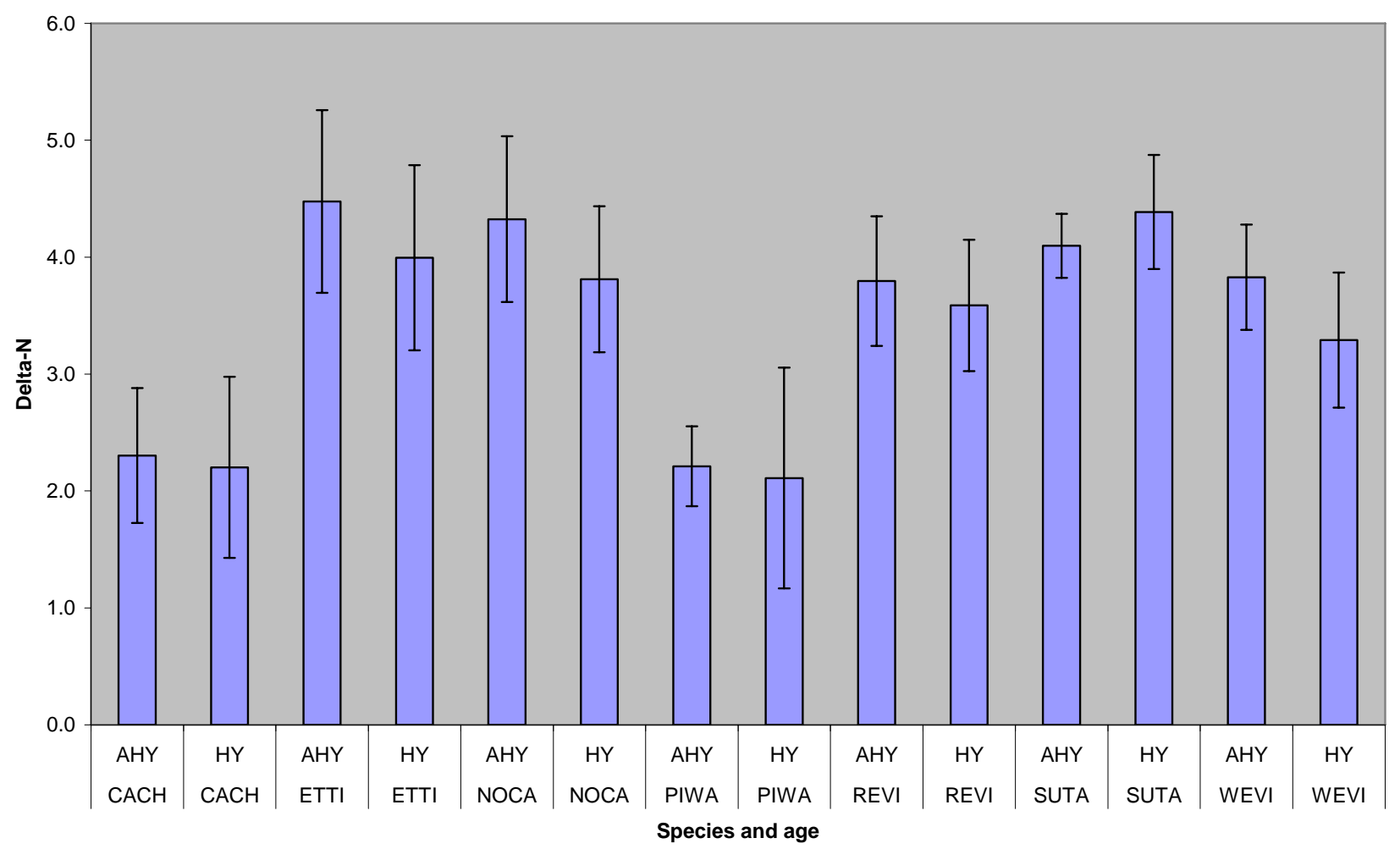


Figure 2. Delta-N values of whole blood sampled from After Hatching Year (AHY or adult) and Hatching Year (HY or fledgling) of seven omnivorous bird species in June and July of 2006 at the Savannah River Site, Aiken, South Carolina. Species designations and statistics as in Figure 1.

delta-N values for blood from AHY and $\mathrm{HY}$ birds

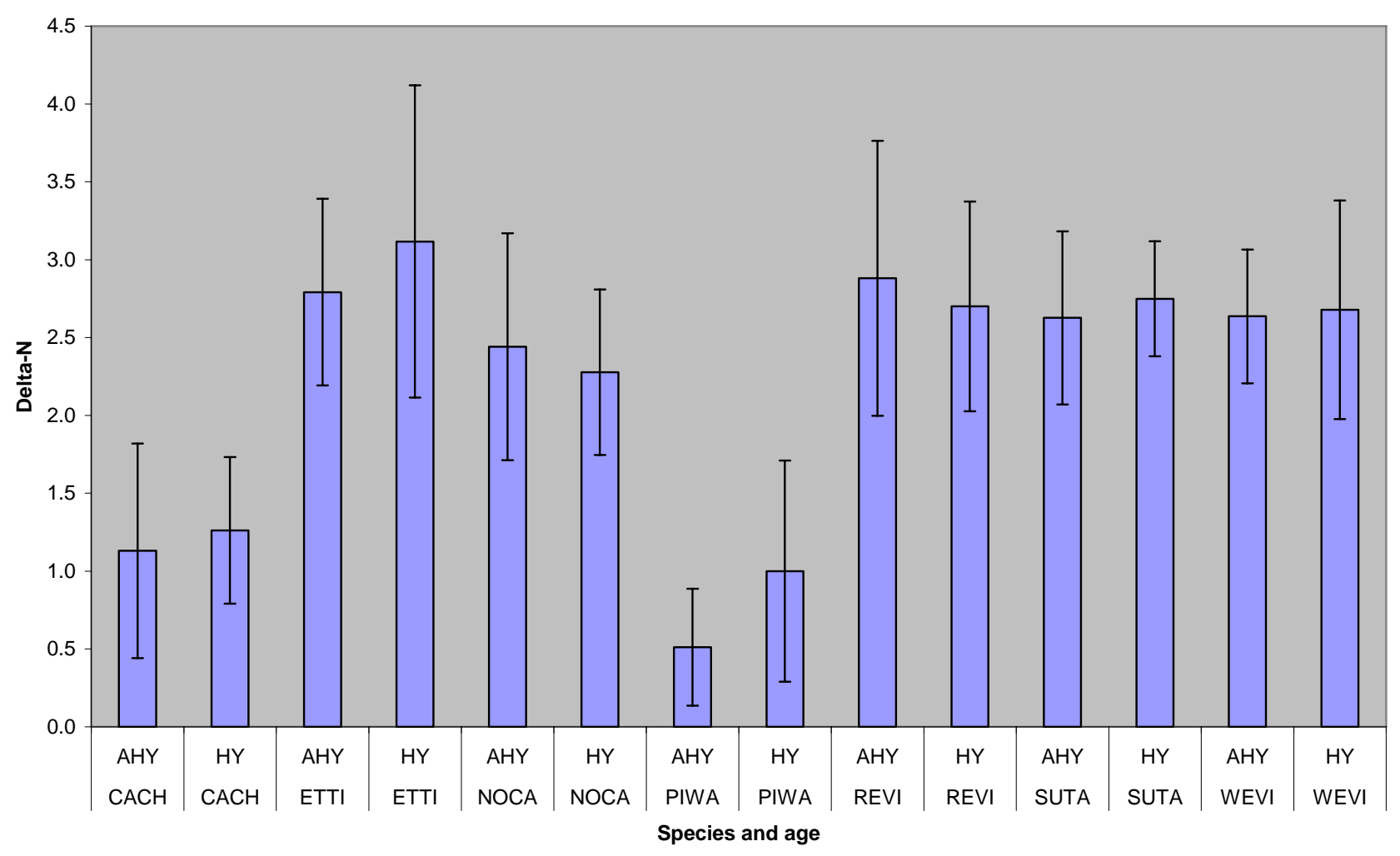


Figure 3. Differences in delta-N between tip and base of tail feathers of seven species of omnivorous HY birds at the Savannah River Station, Aiken SC. Negative values indicate birds were consuming fewer fruit and more insects when their tail feathers were first growing (i.e., when they were younger and fed more frequently by their parents). Error bars represent standard deviations. For no species is the difference in delta-N between tip and base of feather significant $(\mathrm{P}>0.25)$.

delta-N of retrices of HY birds (tip - base)

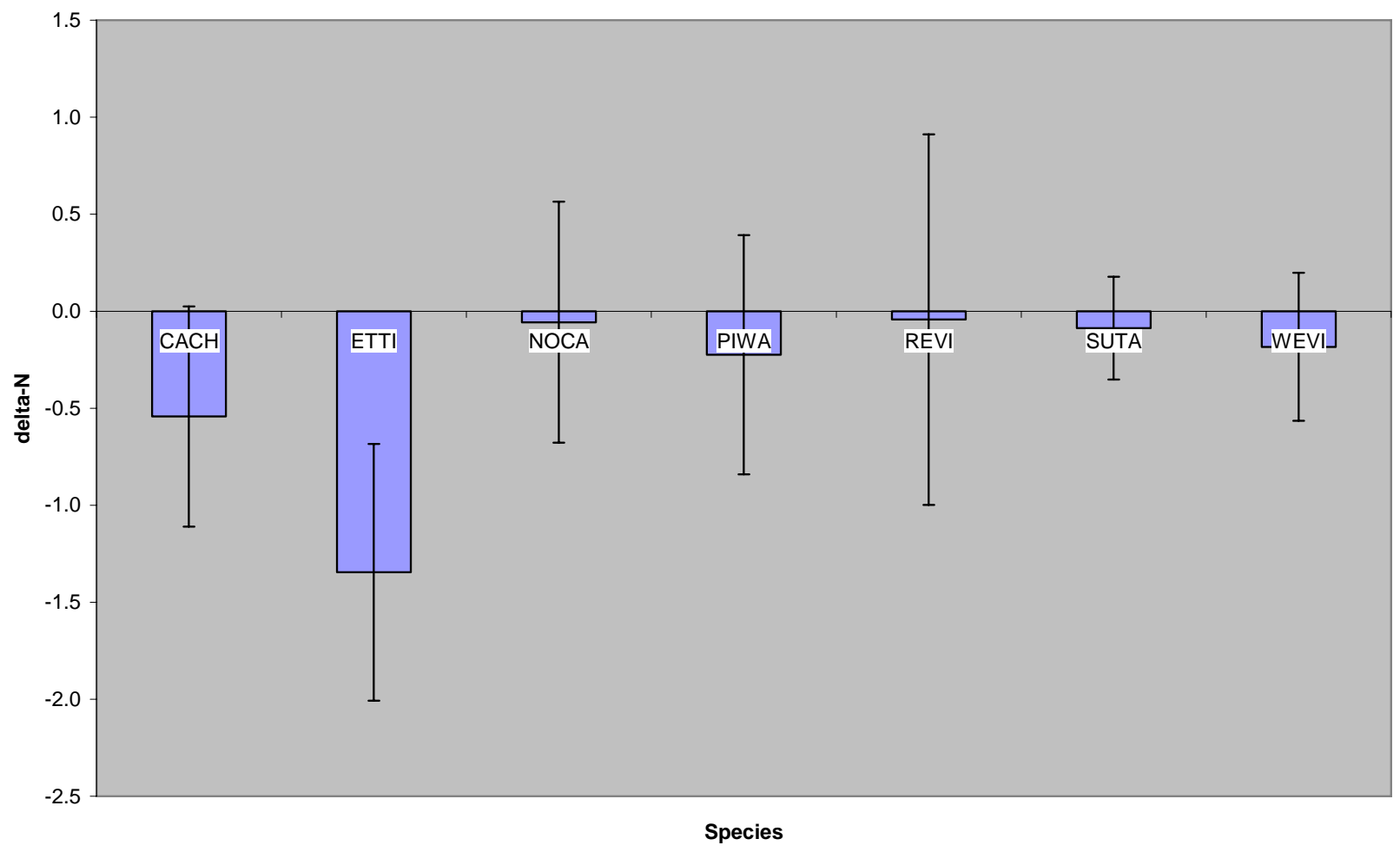




\section{Section 3}

Assessing the Importance of Fruit To Over-wintering Resident and Migrant Birds at SRS

Scott F. Pearson ${ }^{1,2}$, Adam D. Smith ${ }^{3}$, Douglas J. Levey ${ }^{2}$, Scott McWilliams ${ }^{3}$, and Cathryn H. Greenberg ${ }^{4}$

${ }^{1}$ Department of Biology, University of Florida, P.O. Box 118525, Gainesville, Florida 32611, USA

${ }^{2}$ Washington Department of Fish and Wildlife, Wildlife Research Division, 1111 Washington St. SE., Olympia, Washington, 98501, USA

${ }^{3}$ Department of Natural Resources Science, University of Road Island, Kingston, Road Island 02881

${ }^{4}$ U.S. Department of Agriculture, Forest Service, Southern Research Station, Bent Creek Research and Demonstration Forest, Asheville, North Carolina 28806 


\begin{abstract}
Many species of migrant and over-wintering birds switch from a diet dominated by arthropods during the breeding season to one that includes at least some fruits and/or seeds. To assess the importance of fruit resources to migrating and overwintering birds, we tested the following predictions: (1) among species of birds, diets become increasingly divergent (or specialized) in the fall and winter, particularly during the coldest months; (2) importance of fruit to overwintering migrants is greater during the winter when temperatures are colder and arthropod biomass is reduced; and (3) fruit and seed resources contribute significantly to building tissues during the fall and winter months and are therefore important to self-maintenance and survival. For all of our comparisons, we put fruit resources in context with other food types commonly consumed by birds in the fall and winter at our study site in South Carolina.
\end{abstract}

Our stable isotope data indicated a shift from a summer diet composed primarily of invertebrates for all species in the late summer to a more specialized diet in the fall and winter. Some species consumed more fruit, others consumed more grass and rush seeds, and others continued to consume primarily arthropods. As predicted, there was a significant shift for some overwintering migrants (yellow-rumped warbler and ruby-crowned kinglet) to a diet containing more fruit in the winter when arthropod resources were limited and fruit was still relatively abundant. For overwintering seed-eaters, there was a similar shift but to a diet with more grass and rush seeds in the winter. Fecal data independently support the importance of fruit resources to overwintering migrant and resident birds in the fall and winter. 


\section{Introduction}

Results from our long-term study of the temporal and spatial availability of fruit resources and the use of fruits by animals at the Savannah River Site (South Carolina), indicate that some fruitproducing plant species produce significant fruit biomass ( $>1 \mathrm{Kg}$ dry mass of pulp per ha/yr) and that vertebrates consume $\geq 50 \%$ of fruits of many of these species (McCarty et al. 2002). Approximately half of the fruit-producing species produced fruit in the fall and winter, and their fruits were primarily consumed by over-wintering animals (McCarty et al. 2002). Many fruits like those of Morella cerifera ripen in November but peak fruit removal by animals occurs in the coldest months of December and January and mean time to fruit removal is positively correlated with colder winters (Kwit et al. 2004a). Moreover, local abundance of bird species like the hermit thrush (Catharus guttatus) and the yellow-rumped warbler (Dendroica coronata) can be predicted by fruit biomass on these same study plots (Kwit et al. 2004b) suggesting that overwintering species are tracking fruit resources. Experimental manipulations of fruit abundance indicate that over-wintering birds are indeed tracking fruit resources at the local scale and, once discovered, warblers quickly exploit the fruit (Borgamnn et al. 2004). Taken together, these studies suggest that fruit is an important food resource particularly to over-wintering birds at our study site. However, truly evaluating the importance of different food resources is difficult unless one can link the resource to survival and reproduction.

To assess the importance of fruit resources to migrating and overwintering birds, we tested the following predictions: (1) among species of birds, diets become increasingly divergent (or specialized) in the fall and winter, particularly during the coldest months; (2) importance of fruit to over-wintering migrants is greater during the winter when temperatures are colder and arthropod biomass is reduced; and (3) fruit and seed resources contribute significantly to building tissues during the fall and winter months and are therefore important to selfmaintenance and survival. For all of our comparisons, we put fruit resources in context with other available resources consumed by birds in the fall and winter (seeds and arthropods). 
To compare the degree of dietary overlap in the summer, fall and winter we compared the $\mathrm{N}$ and C isotopic signatures of resident and migratory birds during the fall/winter of 1998/1999 and 1999/2000. To assess the relative contribution of fruit, arthropods and grass and rush seeds to building tissues (plasma and whole blood) we used concentration dependent linear mixing models. Independently, we compared the results derived from the mixing models to the percent of these same food resources in the feces collected from the same birds as the isotopes.

\section{Methods}

Study Site. This study was conducted from September 1998 to February 2000 at the Savannah River National Environmental Research Park located in Aiken and Barnwell counties, South Carolina, USA $\left(33^{\circ} 18^{\prime} \mathrm{N}, 8137^{\prime} \mathrm{W}\right)$. This $780 \mathrm{~km}^{2}$ site is located along the Savannah River in the Sandhill and upper Coastal Plain physiographic provinces. Forested areas cover $>80 \%$ of the Site (Workman and McLeod 1990). Most of the forested area consists of mosaic of managed longleaf pine (Pinus palustris) and loblolly pine (P. taeda) stands and, to a lesser extent, upland and bottomland hardwood stands (Odum 1991, White and Gaines 2000). Detailed descriptions of the site, its history, and management are provided by Odum (1991), White and Gaines (2000) and especially in Kilgo and Blake (2005).

Bird capture, sample collection and preparation. Birds were captured from 22 Sept. 1998 - 19 February 1999 and from 24 August 1999 - 5 February 2000 using mist nets. Nets were placed in upland and bottomland clearcuts, bottomland hardwood stands, upland pine stands, and upland hardwood stands. Captured birds were placed in clean cotton cloth bags and held until processed. Processing consisted of weighing each bird and measuring its wing cord, tarsus and culmen, identifying birds to species, and determining the age and sex following Pyle (1997). When possible, approximately $120 \mu \mathrm{l}$ blood sample was collected from the brachial vein of each bird captured. When an adequate sample was obtained, approximately half the blood sample was centrifuged to separate plasma from cells. Plasma and whole blood were analyzed separately. We did not separate the blood if only a small volume of blood was taken because of difficulty obtaining an adequate sample or because of the bird's small size (e.g., ruby-crowned kinglet $\approx$ $6 \mathrm{~g})$. We preferred using plasma for our stable isotope analysis because of its short half life (1-2 
days; Pearson et al. 2003) and therefore information on very recent diet. Blood samples were initially stored at $-70^{\circ} \mathrm{C}$, then freeze-dried. A tail feather was plucked from each bird and initially stored in a plastic bag and later washed in ether to remove oils (Hobson and Clark 1992) and cut into small pieces $\left(\mathrm{ca} 1 \mathrm{~mm}^{3}\right)$. Approximately $1 \mathrm{mg}$ of each feather and blood sample (dry mass) was loaded into tins for isotopic analysis. When provided by the bird, fecal samples were scraped from the inside of the cloth bag used to hold the bird, placed in vials containing $90 \%$ ethyl alcohol. Invertebrates and fruits were collected from the same sites and the same time of year as the birds. Invertebrates were collected by sweeping the vegetation using a butterfly net and by plucking invertebrates from the litter. Seeds were removed from fruit and both Invertebrates and fruit were placed in a drying oven $\left(55^{\circ} \mathrm{C}\right)$ for seven days. Fruits and seeds were then ground into a powder using a mortar and pestle and approximately $1 \mathrm{mg}$ of each sample was loaded into tins for isotopic analysis.

To calculate the percent fruit, invertebrate and seeds in each fecal sample we placed samples into a petri dish, spread the sample apart using tweezers and a spatula and examined it under a dissecting microscope. The sample was placed over a grid pattern and we counted the number of squares obscured by arthropods, fruits, seeds and other items in 4-5 views to derive our estimate of the percent of the cells obscured by each dietary item. We identified many of the arthropods parts to order following the methods of Ralph et al. 1985 and similar to Moorman et al. (2007).

Isotope analysis. The University of California Stable Isotope Facility performed all isotope analyses. Carbon and $\mathrm{N}$ stable isotope ratios were determined on $\mathrm{CO}_{2}$ and $\mathrm{N}_{2}$ gases, produced by combustion of samples in an elemental analyzer coupled to a continuous flow isotope ratio mass spectrometer (ANCA-CFIRMS; Europa Scientific Crewe, England; ANCA combustion unit and 20-20 mass spectrometer). The ratio of stable isotopes is expressed in delta ( ) notation:

$\left[\left(\mathrm{R}_{\text {sample }} / \mathrm{R}_{\text {standard }}\right)^{-1}\right] \mathrm{x} 1,000$, where is the isotope ratio of the sample relative to a standard. For $\mathrm{C}$, the international standard is the Peedee Belemnite (PDM) limestone formation. For N, the standard is atmospheric $N(A I R)$. $R_{\text {sample }}$ and $R_{\text {standard }}$ are the fractions of heavy to light isotopes in the sample and standard, respectively. Isotope ratios were derived by comparison of sample with reference gas injections in each analytical cycle. Raw delta values were normalized to international values ${ }^{15} \mathrm{~N}$ AIR and ${ }^{13} \mathrm{C}$ PDB using combusted standard samples (ammonium 
sulfate + sucrose) inserted at intervals in each batch of samples. Masses of $\mathrm{C}$ and $\mathrm{N}$ in each sample were derived by integrating the ion beam intensities and comparing the integrals to those of standard samples with known concentrations of $\mathrm{C}$ and $\mathrm{N}$. Carbon and $\mathrm{N}$ mass were converted to percent by dividing the mass of $\mathrm{N}$ and $\mathrm{C}$ in the sample by the sample weight and multiplying by 100 .

Defining fall and winter. To compare diet contribution to tissues and fecal content between relatively mild fall conditions and the colder winter conditions for each of the two falls/winters, we created cumulative sum graphs (Page 1961) of the daytime high temperatures from the Savannah River Site. Cumulative sum graphs are a visual way of identifying systematic changes in states over time (e.g., from warm to cold). Cumulative sums are the differences between the values (e.g., daily temperatures) and the average for the period of interest. These differences sum to zero so the cumulative sum always ends at zero. When the slope is increasing on the graphs the temperature was above average for the overall period and when it is decreasing it was below average. The peak is when the change state occurs from a warmer than average to a colder than average. To identify this peak in the graph or the "change state", we used the change point analysis approach and software developed by Taylor (2000).

Mixing models. We used concentration-dependent linear mixing models to estimate the isotopic contribution of food sources to avian tissues. The SIAR program (Parnell et al. 2008), operated within the R statistical computing environment ( $R$ development core team 2007), uses a Bayesian approach to find the most probable contribution of dietary sources to tissues by relating the isotopic composition of a tissue to the isotopic composition of possible food sources while accounting for the elemental concentrations and tissue-specific discrimination factors of these food sources. The Bayesian approach offers the advantage of propagating uncertainty in the isotopic values of sources and discrimination factors, thus resulting in robust probability distributions for estimated dietary proportions.

We related $\delta 15 \mathrm{~N}$ and $\delta 13 \mathrm{C}$ in songbird blood plasma or whole blood (Ruby-crowned Kinglets only) to $\delta 15 \mathrm{~N}$ and $\delta 13 \mathrm{C}$ in possible fruit, arthropod and seed sources (i.e., dietary endpoints), using source-specific $\mathrm{N}$ and $\mathrm{C}$ elemental concentrations and discrimination factors. We 
estimated dietary contributions for a sample of at least five individuals of a given species within a given season. Although SIAR can estimate dietary contributions at the level of the individual, our unpublished simulations of dietary contributions to individual and groups of individuals consuming known diets exhibited exceedingly variable results at group sizes of three individuals or less. We found that the default number of burn-in iterations $(50,000)$, subsequent iterations $(150,000)$, and thinning interval (15 iterations) worked satisfactorily for our data, and resulted in probability distributions for estimated dietary proportions based on 10,000 iterations. We also retained the default setting of uniform priors for dietary contributions.

To estimate the probability that the contribution of a particular dietary endpoint exceeds that of another within or between seasons, we compared the mixing model output on an iteration by iteration basis (A. Parnell, pers. comm.). For example, to find the probability that the contribution of fruit in the winter diet of Ruby-crowned Kinglets exceeds that of fruit in their fall diet, we divided the number of iterations in which the proportion of winter fruit is greater than the proportion of fall fruit (i.e., 8399) by the total number of iterations (i.e., 10,000), which in this case produces a probability of 0.8399 . A probability of 0.5 indicates no difference in dietary contributions. Probabilities are symmetrical around 0.5 , so that a probability of 0.75 is the same as a probability of 0.25 .

Defining dietary endpoints. We identified appropriate arthropod, fruit, and seed taxa to serve as dietary endpoints based on personal observation of foraging birds at Savannah River Site and a literature review. Consequently, the combination of dietary endpoints used in mixing models varied among species (Table $1 \& 2$ ). Specifically, we identified seven potential dietary endpoints: fruit, high- and low- trophic level arthropods from $\mathrm{C} 3(\delta 13 \mathrm{C}<-21 \%)$ and $\mathrm{C} 4(\delta 13 \mathrm{C}$ $>-18 \%$ ) environments, and C3 and C4 seeds (Table 2). We applied a k-means cluster analysis (Steinley 2006) to the entire arthropod dataset, which identified two groups of arthropods separated at a $\delta 15 \mathrm{~N}$ of $3 \%$; we classified those above and below this cutoff as high- and lowtrophic arthropods, respectively. To maximize the use of our arthropod dataset, we calculated $\delta 13 \mathrm{C}$ values for $\mathrm{C} 3$ and $\mathrm{C} 4$ arthropods across trophic levels. Likewise, we calculated $\delta 15 \mathrm{~N}$ values for high- and low-trophic arthropods independent of C3 or C4 status. For example, lowtrophic $\mathrm{C} 4$ arthropods were assigned the average $\delta 13 \mathrm{C}$ value of all $\mathrm{C} 4$ arthropods, regardless of 
trophic status, and the average $\delta 15 \mathrm{~N}$ value of all low-trophic arthropods, regardless of C source. This method produced more consistent $\delta 13 \mathrm{C}$ and $\delta 15 \mathrm{~N}$ values among dietary endpoints. We used a similar approach to calculate seed $\delta 15 \mathrm{~N}$ endpoint calculations. That is, we assigned seeds the average $\delta 15 \mathrm{~N}$ value for all seeds regardless of C source. Small sample size in C3 seeds resulted in an unrealistically low standard deviation in $\delta 13 \mathrm{C}$ values, so we scaled the standard deviation to produce a coefficient of variation equivalent to that in $\mathrm{C} 4$ seeds. For fruits and seeds, we weighted (using sample size) the contribution from a given taxon when calculating average $\delta 15 \mathrm{~N}$ and $\delta 13 \mathrm{C}$ values, but used the uncertainty associated with the overall averages. For arthropods, each sample represented a different taxon (although often within the same order), so we elected to calculate unweighted averages for $\delta 15 \mathrm{~N}$ and $\delta 13 \mathrm{C}$.

Dietary discrimination values. We estimated arthropod and fruit diet discrimination values $(\Delta)$ using our experimentally derived equations relating diet-tissue discrimination values to the elemental concentration of the diet (Table 3; Pearson et al. 2003). For plasma and whole blood, we calculated $\Delta \mathrm{N}$ and $\Delta \mathrm{C}$ for the fruit endpoint based on the average $\mathrm{N}$ and $\mathrm{C}$ concentrations of all fruit samples, and for arthropod endpoints based on the average $\mathrm{N}$ and $\mathrm{C}$ concentrations of all arthropod samples (Table 2). We calculated the uncertainty (standard deviations) associated with $\Delta \mathrm{N}$ and $\Delta \mathrm{C}$ by summing in quadrature the standard deviation of tissue-specific regression residuals from the published equations and the standard deviation of discrimination estimates resulting from uncertainty in the elemental concentrations of the dietary endpoints. That is, the change in $\Delta \mathrm{N}$ or $\Delta \mathrm{C}$ when using $\mathrm{N}$ and $\mathrm{C}$ concentrations one standard deviation above or below their average represented the standard deviation of the discrimination value due to uncertainty in elemental concentrations. Similarly, we calculated plasma $\delta 15 \mathrm{~N}$ and $\delta 13 \mathrm{C}$ discrimination values and their uncertainty for the seed endpoint based on the average $\mathrm{C}$ and $\mathrm{N}$ concentrations of all seed samples (Table 2). Although not used in any mixing models, estimated $\Delta$ values for whole blood from the Pearson et al. (2003) equations agreed generally with published estimates of discrimination in blood cells of birds on a corn diet (Carleton and Martinez del Rio 2005).

Statistical comparison of dietary endpoints. We conducted a multivariate analysis of variance (MANOVA; PROC GLM; Version 9.1, SAS Institute Inc. 2002) to determine whether $15 \mathrm{~N}$ and $13 \mathrm{C}$ varied among dietary endpoints. MANOVA indicated that dietary endpoints differed in a 
multivariate fashion (see results), thus we used Scheffe's multiple contrasts within follow-up univariate ANOVAs to establish how $\delta 15 \mathrm{~N}$ and $\delta 13 \mathrm{C}$ differed among dietary endpoints. Because PROC GLM cannot handle missing data, MANOVA results are based on a reduced endpoint dataset such that only dietary samples with measures of $\delta 15 \mathrm{~N}$ and $\delta 13 \mathrm{C}$ are used in the calculation of MANOVA test statistics; we conducted followup ANOVAs and pairwise contrasts in PROC MIXED (SAS Institute Inc. 2002) using maximum likelihood estimation so that statistical comparisons are based on the full $\delta 15 \mathrm{~N}$ and $\delta 13 \mathrm{C}$ datasets of dietary endpoints (i.e., those used in the mixing model analysis).

Tissue isotopic comparison. For feather base $\delta 15 \mathrm{~N}$ and $\delta 13 \mathrm{C}$ values (i.e., most recent summer growth), we applied a discrimination value based on the Pearson et al (2003) equation for feathers assuming a 100\% arthropod diet (Table 3), using the average $\mathrm{N}$ and $\mathrm{C}$ concentrations for all arthropod samples (Table 2). For fall and winter values, we assumed a mixed diet (tissue $\mathrm{N}$ and $\mathrm{C}$ representing $75 \%$ arthropod, and $25 \%$ fruit or seed sources, depending on the species); thus, we calculated the appropriate tissue discrimination value based on the weighted average of the discrimination values for a 100\% arthropod diet and a 100\% fruit or seed diet (Table 3).

\section{Results}

Defining fall and winter. For the 1998-1999 season, our change state analysis indicated that the daily high temperature shifted from relatively mild fall conditions to cooler winter conditions on 8 Dec. $1998(95 \% \mathrm{CI}=24 \mathrm{Nov}$. and 31 Jan.). There is 100\% confidence that a change actually occurred. For the 1999-2000 season, the transition occurred on 12 November $1999(95 \% \mathrm{CI}=$ 19 Sept. and 29 January). There is $93 \%$ confidence that a change state occurred. For subsequent analyses, we use these transition dates to define fall ( $<8$ Dec. in 1998 and $<12$ November in 1999) and winter ( $\geq 8$ Dec. in 1998, and $\geq 12$ November in 1999). The average daily high 1998

temperature prior to the change state was $26.9^{\circ} \pm 4.6^{\circ}$ (fall) and $17.5^{\circ} \pm 6.4^{\circ}$ after (winter). The average daily high 1999 temperature prior to the change state was $27.0^{\circ} \pm 5.8^{\circ}$ (fall) and $14.6^{\circ} \pm$ $6.1^{\circ}$ after (winter).

Birds captured and samples collected. Over the two fall/winters, we captured 653 birds of 47 species. Yellow-rumped warbler and ruby-crowned kinglet represented $24 \%$ and $15 \%$ of the 
birds captured respectively. Eighteen species were captured $<5$ times and are not included in any of the following analyses. Table 1 provides a list of species and their scientific names included in the following analyses.

Are fruit resources an important component of feces during the fall and winter months? We collected 288 fecal samples from bird 33 species. The yellow-rumped warbler and ruby-crowned kinglet dominated the samples and represented $36 \%$ and $22 \%$ of the total samples. Twenty of these 33 bird species (61\%) ate at least some fruit. Fecal samples from nine species contained no fruit but only two of these nine species had sample sizes greater or equal to 10 (white-throated sparrow and dark-eyed junco). In Table 4 we present results from species with $\geq 4$ fecal samples per season and where we also have adequate plasma or blood samples for isotopic analyses. American robin, hermit thrush, and yellow-rumped warbler, all species previously described as being highly frugivorous during the fall and winter months, consistently had greater than $70 \%$ fruit pulp in their feces (Table 4). Of the fecal samples where fruit was identified, Morella cerifera was the only fruit found in yellow-rumped warbler feces and in all but one rubycrowned kinglet feces. One ruby-crowned kinglet feces contained both Ilex opaca and Morella cerifera and one only contained only Toxicodendron radicans. All American robin feces contained only one fruit species, Ilex opaca. Hermit thrush feces with fruit contained Ilex opaca (4 of 5) and one contained both Toxicodendron radicans and Euonymus americanus. Four tufted titmouse feces contained Ilex opaca, 1 contained Toxicodendron radicans and one contained an unknown fruit species. Gray catbird feces contained Phytolaaca americana, Morella cerifera, and Toxicodendron radicans. Northern cardinal feces contained Ilex opaca and Morella cerifera. The eastern towhee feces contained the pulp and skin from an unknown fruit species. Grass and rush seed parts (highly fragmented) in feces were not identified to species but we did observed white-throated sparrows consuming Phragmites communis, Scirpus cyperinus and an unidentified Paspalum spp. and we observed dark-eyed juncos consuming species of Poa spp. and Andropogon spp. on the same sites where they were captured (all were collected for deriving endpoints). Because arthropod parts were extremely fragmented, there were clearly biases associated with the orders that we could identify (e.g., spider fangs are easy to identify), and because we could not consistently identify invertebrate parts in feces to order, we do not attempt to quantify insect parts by order but simply quantified the percent insect observed in the feces. We did however, observe spider fangs in the feces of most species and we observed parts of 
Dipteran, Hymenopteran, Coleopteran, and occasionally Hemipteran, and lepidopteran larvae mandibles. In addition, we observed insect egg casings in both ruby-crowned kinglet and yellow-rumped warbler feces - especially during the winter months.

Do dietary end points differ? Dietary endpoints differed multivariately in $\delta 15 \mathrm{~N}$ and $\delta 13 \mathrm{C}$ (MANOVA: Wilk's Lambda $=0.011, \mathrm{~F} 12,78=55.97, \mathrm{P}<0.0001$ ), and univariate ANOVAs indicated differences among dietary endpoints in $\delta 15 \mathrm{~N}$ (ANOVA: F6,76 $=40.18, \mathrm{P}<0.0001$ ) and $\delta 13 \mathrm{C}$ (ANOVA: F6,65 $=105.71, \mathrm{P}<0.0001$ ); pairwise contrasts between all dietary endpoints are summarized in Figure Table 2.

Do diets become more specialized (divergent) among species in the fall and winter? Because tail feathers are molted at the end of the nesting season (late summer) for all of the species included in this study, it appears that there is broad overlap in late summer diet (Figure 1) and the enriched (more positive) $\delta 15 \mathrm{~N}$ signature suggests that arthropods likely contribute significantly to the composition of their feathers. During the fall and winter, there appears to be divergence in both $\delta 15 \mathrm{~N}$ and $\delta 13 \mathrm{C}$ signature of plasma or blood among bird species suggesting divergence in diet (Figure 1) or at least the dietary contribution to these tissues. This depleted (less positive) $\delta 15 \mathrm{~N}$ signature suggests lower trophic contributions to these tissues in the fall and winter than summer which is consistent with fewer arthropods in the diet. For the dark-eyed junco and white-throated sparrow, plasma is $\delta 13 \mathrm{C}$ was enriched (less negative) in the winter suggesting an increase in the contribution of $\mathrm{C} 4$ dietary items to these tissues, a result consistent with a diet consisting of more seeds and fewer arthropods during the winter.

Do fruit and seed resources contribute significantly to building tissues during the fall and winter months? For the two overwintering migrants with adequate sample sizes for inter-seasonal comparisons, yellow-rumped warbler and ruby-crowned kinglet, there was greater contribution of fruit resources to tissues in the winter than the fall as predicted (Table 4, all probabilities $\geq$ 0.77). Average fruit contribution for ruby-crowned kinglets and yellow rumped warblers in the fall was $4.3 \%$ and $10.2 \%$ respectively and winter contribution was $18.2 \%$ and $36.8 \%$ in $1998 / 19.2 \%$ in 1999. In addition, there was greater contribution of low trophic arthropods and less contribution of high trophic arthropods in the winter than the fall (Table 4, all probabilities $\geq$ 0.77 or $\leq 0.16$ ). For the yellow-rumped warbler, we predicted that fruit contribution to tissues would be greater during the colder winter of 1999/2000 (1998/1999 Ave. high temperature = 
$17.5^{\circ} \pm 6.4^{\circ} \mathrm{SD}, 1999 / 2000$ Ave. high temperature $\left.=14.6^{\circ} \pm 6.1^{\circ} \mathrm{SD}\right)$, but this was not the case (Table 4). During this colder winter of 1999/2000, there was a concomitant increase in the contribution of high trophic arthropods (most likely spider, based on fecal analysis) to yellowrumped warbler plasma. For our over-wintering seed eater, for the white-throated sparrow, there was greater contribution of seeds during the winter than the fall (Table 4) when seeds contributed $35.6 \%$ on average to white-throated sparrow plasma. For our one resident species, the northern cardinal, there was a greater contribution of fruit in the fall and a greater contribution of seeds during the winter (Table 4). Fruit contributed $25.8 \%$ in fall/16.0\% in winter and seed contributed $32.3 \%$ in the winter.

\section{Discussion}

Stable isotope data indicate a shift from a summer diet composed primarily of invertebrates for all species in the late summer to a more specialized diet in the fall and winter (Figure 1) when, some species consume more fruit, others consume more grass and rush seeds, and others continue to consume primarily arthropods. As predicted, there was a significant shift for the more frugivorous over-wintering migrants (yellow-rumped warbler and ruby-crowned kinglet) from a diet dominated by invertebrates during the relatively warm fall to a diet with a significant component of fruit when invertebrate resources were limited and fruit was still relatively abundant. For overwintering seed eaters, there was a similar shift but to a diet with more grass and rush seeds. Other species continued to primarily consume arthropods during the winter. However, we emphasize that our mixing models estimate the contribution of dietary end points to the isotopic composition of a tissue. It does not estimate the fraction of a source in the animals diet (Martinez del Rio et al. 2009). Fecal data independently support also indicate the importance of fruit resources to birds in the fall and winter.

As pointed out by Martinez del Rio et al. 2009, the contribution of dietary end points to a tissue may not equal the fraction of a source in an animal's diet because: (1) the C:N ratio of various sources may differ, (2) the assimilation efficiency of elements may differ among sources, (3) diet-tissue discrimination, and (4) isotopic routing. We address items 1 and 2 by using concentration-dependent mixing models (Phillips and Koch 2002). This is especially important 
because we know that the isotopic concentrations differ considerably between fruit and invertebrates (see Pearson et al. 2003). We address 3 by using our experimentally derived discrimination values (see Pearson et al 2003).

The rate at which animals incorporate the isotopic signal of their diets into their tissues varies among organisms and among tissues within a single individual. To address this variation, we used birds relatively similar in size and, for the most part, only compared the same tissues or used tissues with relatively short half lives (e.g. plasma).

A possible explanation for the difference in the apparent increase in the contribution of fruit to tissues in the winter could simply be the result of differences in incorporation rates when metabolic rates are increased during the colder winter months. However, experimental evidence from house sparrows (Passer domesticus) indicates that cold exposure only has no effect on ${ }^{15} \mathrm{~N}$ incorporation rate and a small and biologically insignificant effect on ${ }^{13} \mathrm{C}$ incorporation rate (Carleton and Martinez del Rio 2005).

Conservation implications - Forest management depending on type and extent can positively or negatively affect the biomass of fruit and seed resources. Maintenance of pin savannah habitats or the creation of gaps in upland and bottomland hardwood stands generally increases fruit and seed abundance. In addition, longer intervals between prescribed surface fires in some upland pine stands can increase fruit biomass (Borgmann et al. 2004).

\section{Acknowledgments}

This project was supported by funds (Grant \#SRS 33-CA-99-523) provided by the Department of Energy - Savannah River Operations Office through the Forest Service Savannah River and Forest Service Southern Research Station, Asheville, North Carolina under Interagency Agreement DE-IAS09-76SR00056. We thank J. Blake and J. Kilgo for helping with logistics and for creating a friendly research environment. We thank Kathy Borgmann, Alicia Brinton, and Colette DeGrady for field assistance and the Savannah River Ecology Lab for library and computer access. Co-authors on this manuscript are: Scott Pearson (lead author), Adam Smith, and Scott McWilliams. 


\section{References}

Borgmann, K.L., S.F. Pearson, D.J. Levey, and C. H. Greenberg. 2004. Wintering yellowrumped warblers (Dendroica coronata) track manipulated abundance of Myrica cerifera fruits. The Auk 121:74-87.

Carleton, S.A. and C. Martinez del Rio. 2005. The effect of cold-induced increased metabolic rate on the rate of ${ }^{13} \mathrm{C}$ and ${ }^{15} \mathrm{~N}$ incorporation in house sparrows (Passer domesticus). Oecologia 144:226-232.

Hobson , K.A., and R.G. Clark. 1992. Assessing avian diets using stable isotopes II. Factors influencing diet-tissue fractionation. Condor 94:189-197.

Kilgo, J.C., and J.I. Blake, eds. 2005. Ecology and management of a forested landscape: Fifty years on the Savannah River Site. Island Press, Washington D.C.

Kwit, C., D.J. Levey, C.H. Greenberg, S.F. Pearson, J.P. McCarty, and S. Sargent. 2004. Cold temperature increases winter fruit removal rate of a bird-dispersed shrub. Oecologia 139:30-34.

Kwit, C., D.J. Levey, C.H. Greenberg, S.F. Pearson, J.P. McCarty, S. Sargent, and R. Mumme. 2004. Fruit abundance and local distribution of wintering hermit thrushes (Catharus guttatus) and yellow-rumped warblers (Dendroica coronate) in South Carolina. The Auk 121:46-57.

Levey, D. J. and F. G. Stiles. 1994. Evolutionary precursors of long-distance migration: resource availability and movement patterns in neotropical landbirds. American Naturalist 140:447-476.

Martinez del Rio, C., N. Wolf, S.A. Carleton, and L.Z. Gannes. 2009. Isotopic ecology ten years after a call from more laboratory experiments. Biological Reviews 84:91-111. 
McCarty, J.P., D.J. Levey, C.H. Greenberg, and S. Sargent. 2002. Spatial and temporal variation in fruit use by wildlife in a forested landscape. Forest Ecology and Management 164: 277-291.

Moorman, C.E., L.T. Bowen, J.C. Kilgo, C.E. Sorenson, J.L. Hanula, S. Horn, and M.D. Ulyshen. 2007. Seasonal diets of insectivorous birds in canopy gaps in a bottomland forest. Journal of Field Ornithology 78:11-20.

Odum, E.P. 1991. The Savannah River Site as a national environmental park. Pages 79-85 in Integrated Environmental Management (J. Cairns and T.V. Crawford, Eds.). Lewis Publishers, Chelsea, Michigan.

Page, E.S. 1961. Cumulative sum control charts. Technometrics 3:1-9.

Ralph, C.P., S.E. Nagata, and C.J. Ralph. 1985. Analysis of droppings to describe diets of small birds. Journal of Field Ornithology 56:165-174.

Pearson, S.F., D.J. Levey, C.H. Greenberg and C. Martinez del Rio. 2003. Effects of elemental composition on the incorporation of dietary nitrogen and carbon isotopic signatures in an omnivorous songbird. Oecologia 135:516-523.

Parnell, A., R. Inger, S. Bearhop, and A. L. Jackson. 2008. SIAR: Stable Isotope Analysis in R. http://cran.r-project.org/web/packages/siar/index.html

Phillips, D.L., and P.L. Koch. 2002. Incorporating concentration dependence in stable isotope mixing models. Oecologia 130:114-125.

Pyle, P. 1997. Identification guide to North American birds: Part I Columbidae to Ploceidae. Slate Creek Press, Bolinas, California. 
R development core team. 2007. R: a language and environment for statistical computing. Vienna: R Foundation for Statistical Computing. Accessible at $<$ http://www.Rproject.org $>$.

Steinley, D. 2006. K-means clustering: a half-century synthesis. British Journal of Mathematical and Statistical Psychology 59:1-34.

Taylor, W. 2000. Change-Point Analyzer 2.0 shareware program. Taylor Enterprises, Libertyville, Illinois. Web: http://www.variation.com/cpa

White, D.L., and K.F. Gaines. 2000. The Savannah River Site: Site description, land use and management history. Studies in Avian Biology 21:8-17.

Workman, S.W., and K.W. McLeod. 1990. Vegetation of the Savannah River Site: Major community types. Savannah River Site, National Environmental Research Park Program, Aiken, South Carolina. 
Table 1. Dietary endpoints used in the construction of mixing models to estimate the isotopic contribution of food sources to avian tissues. The endpoints used for each species was based on personal observation of foraging birds at Savannah River Site and a literature review. The $\mathrm{N}$ and $\mathrm{C}$ isotopic and elemental composition of dietary endpoints are presented in Table 2.

\begin{tabular}{|c|c|c|c|c|c|c|c|c|}
\hline Species & & $\begin{array}{l}\text { Migratory } \\
\text { Status }\end{array}$ & Fruits & $\begin{array}{l}\text { Arthropods (high } \\
\left.\qquad-\mathrm{C}_{3}\right)\end{array}$ & $\begin{array}{l}\text { Arthropods (low } \\
\left.\qquad-C_{3}\right)\end{array}$ & $\begin{array}{l}\text { Arthropods (high } \\
\left.\qquad-\mathrm{C}_{4}\right)\end{array}$ & $\begin{array}{l}\text { Arthropods (low } \\
\left.\qquad-\mathrm{C}_{4}\right)\end{array}$ & $\begin{array}{c}\text { Seeds } \\
\left(\mathrm{C}_{3}\right)\end{array}$ \\
\hline American Robin & $\begin{array}{l}\text { Turdus } \\
\text { migratorius }\end{array}$ & $\mathrm{O}^{\mathrm{b}}$ & $x$ & $x$ & $x$ & & & \\
\hline Dark-eyed Junco & Junco hyemalis & $\mathrm{O}$ & & $x$ & $x$ & $x$ & $x$ & $x$ \\
\hline Eastern Towhee & $\begin{array}{l}\text { Pipilo } \\
\text { erythrophthalmus }\end{array}$ & $\mathrm{R}$ & $x$ & $x$ & $x$ & $x$ & $x$ & $x$ \\
\hline Gray Catbird & $\begin{array}{l}\text { Dumetella } \\
\text { carolinensis }\end{array}$ & $\mathrm{M}^{\mathrm{c}}$ & $x$ & $x$ & $x$ & & & \\
\hline Hermit Thrush & Caltharus guttatus & $\mathrm{O}$ & $x$ & $x$ & $x$ & & & \\
\hline Northern Cardinal & $\begin{array}{l}\text { Cardinalis } \\
\text { cardinalis }\end{array}$ & $\mathrm{R}$ & $x$ & $x$ & $x$ & $x$ & $x$ & $x$ \\
\hline Ruby-crowned Kinglet $^{a}$ & Regulus calendula & $\mathrm{O}$ & $x$ & $x$ & $x$ & & & \\
\hline Swainson's Thrush & $\begin{array}{l}\text { Caltharus } \\
\text { ustulatus }\end{array}$ & M & $x$ & $x$ & $x$ & & & \\
\hline Tufted Titmouse & $\begin{array}{l}\text { Baeolophus } \\
\text { bicolor }\end{array}$ & $\mathrm{R}$ & $x$ & $x$ & $x$ & & & $x$ \\
\hline White-throated Sparrow & $\begin{array}{l}\text { Zonotrichia } \\
\text { leucophrys }\end{array}$ & $\mathrm{O}$ & & $x$ & $x$ & $x$ & $x$ & $x$ \\
\hline Yellow-rumped Warbler ${ }^{\mathrm{a}}$ & $\begin{array}{l}\text { Dendroica } \\
\text { coronata }\end{array}$ & $\mathrm{O}$ & $\mathrm{X}$ & $x$ & $x$ & & & \\
\hline
\end{tabular}


${ }^{a}$ fruit endpoint based only on Myricaceae (Wax myrtle, Morella cerifera)

${ }^{b}$ Nests in the region but not in our study area. Suspect that these birds are migrants

${ }^{\mathrm{c}}$ Rarely nests in the region. Common during migration. 
Table 2. Isotopic and elemental composition of dietary endpoints used in the construction of mixing models to estimate the isotopic contribution of food sources to avian tissues. Values are presented as mean (standard deviation). See text for specifics of $\delta^{15} \mathrm{~N}$ and $\delta^{13} \mathrm{C}$ for dietary endpoints.

\begin{tabular}{|c|c|c|c|c|c|c|c|}
\hline Dietary endpoint & $\mathrm{n}^{\mathrm{a}}$ & $\delta^{15} \mathrm{~N}(\%)$ & $\% \mathrm{~N}$ & $\delta^{13} \mathrm{C}(\%)$ & $\% \mathrm{C}$ & $\begin{array}{l}\text { Significance } \\
\text { test }{ }^{\mathrm{C}} \delta^{15} \mathrm{~N}\end{array}$ & $\begin{array}{l}\text { Significance } \\
\text { test }^{\mathrm{C}} \delta^{15} \mathrm{~N}\end{array}$ \\
\hline Fruits & $11 / 11$ & $-2.25(2.00)$ & $0.80(0.14)$ & $-29.91(1.23)$ & $50.70(1.48)$ & $\mathrm{C}$ & $\mathrm{A}$ \\
\hline Fruits (Myricaceae) ${ }^{\mathrm{b}}$ & $5 / 5$ & $-2.25(0.28)$ & $0.85(0.20)$ & $-30.02(1.24)$ & $56.08(1.36)$ & $\mathrm{C}$ & A \\
\hline Arthropods (high $\mathrm{C}_{3}$ ) & $15 / 22$ & $5.23(1.30)$ & $10.83(2.33)$ & $-26.02(2.21)$ & $48.16(3.95)$ & A & B \\
\hline Arthropods (low $\mathrm{C}_{3}$ ) & $10 / 22$ & $1.08(0.95)$ & $10.83(2.33)$ & $-26.02(2.21)$ & $48.16(3.95)$ & B & B \\
\hline Arthropods (high $\mathrm{C}_{4}$ ) & $15 / 3$ & $5.23(1.30)$ & $10.83(2.33)$ & $-14.90(2.78)$ & $48.16(3.95)$ & A & $\mathrm{C}$ \\
\hline Arthropods (low $\mathrm{C}_{4}$ ) & $10 / 3$ & $1.08(0.95)$ & $10.83(2.33)$ & $-14.90(2.78)$ & $48.16(3.95)$ & B & $\mathrm{C}$ \\
\hline Seeds $\left(C_{3}\right)$ & $11 / 2$ & $1.43(1.99)$ & $2.65(1.25)$ & $-27.04(0.45)$ & $47.80(2.02)$ & $\mathrm{B}$ & $\mathrm{A}, \mathrm{B}$ \\
\hline Seeds $\left(C_{4}\right)$ & $11 / 9$ & $1.43(1.99)$ & $2.65(1.25)$ & $-12.71(0.21)$ & $47.80(2.02)$ & B & $\mathrm{C}$ \\
\hline
\end{tabular}

aindicates sample size used to calculate $\delta^{15} \mathrm{~N} / \delta^{13} \mathrm{C}$, respectively; $\% \mathrm{~N}$ and $\% \mathrm{C}$ in fruits, arthropods, and seeds calculated from 11,25 , and 11 samples, respectively

${ }^{b}$ fruit endpoint based only on Myricaceae (Wax myrtle, Morella cerifera) used for Yellow-rumped Warblers and Ruby-crowned Kinglets

'Letters differ at $\mathrm{p}<0.001$ (Scheffe's adjusted) 
Table 3. Tissue- and diet-specific discrimination $(\Delta)$ values used in mixing models for estimating the isotopic contribution of food sources to avian tissues. We estimated arthropod and fruit diet discrimination values using published equations relating diet-tissue discrimination values to the elemental concentration of the diet (Pearson et al. 2003). Values are presented as mean (standard deviation). See text for specifics of $\Delta \mathrm{N}$ and $\Delta \mathrm{C}$ calculation.

\begin{tabular}{|c|c|c|c|c|c|c|}
\hline \multirow[b]{2}{*}{ Dietary endpoint } & \multicolumn{3}{|c|}{$\Delta \mathrm{N}(\%)$} & \multicolumn{3}{|c|}{$\Delta \mathrm{C}(\%)$} \\
\hline & Feather & Blood & Plasma & Feather & Blood & Plasma \\
\hline Fruits $^{\mathrm{b}}$ & - & $1.15(0.13)$ & $2.32(0.23)$ & - & $1.41(0.56)$ & $-0.07(0.51)$ \\
\hline Arthropods & $3.77(0.23)$ & $3.04(0.56)$ & $3.20(0.42)$ & $3.07(0.83)$ & $0.64(1.33)$ & $-0.55(0.98)$ \\
\hline Seeds & - & $1.50(0.38)$ & $2.48(0.34)$ & - & $0.54(0.75)$ & $-0.61(0.62)$ \\
\hline
\end{tabular}


Table 4. Estimated fruit composition of feces (\%) and estimated isotopic contribution (\%) of food sources to avian tissues based on linear mixing models. Median values are presented, along with the $95 \%$ frequentist confidence interval (fecal fruit) or Bayesian confidence intervals (mixing model output). First $\mathrm{N}$ value $=$ isotopic contribution sample size and second $\mathrm{N}$ value $=$ estimated contribution of fruit in feces.

\begin{tabular}{|c|c|c|c|c|c|c|c|c|c|}
\hline Species & $\mathrm{N}$ & Fecal fruit & Fruits & $\begin{array}{l}\text { Arthropods } \\
\text { (total) }\end{array}$ & Seeds (total) & $\begin{array}{l}\text { Arthropods } \\
\left(\text { high }-\mathrm{C}_{3}\right)\end{array}$ & $\begin{array}{c}\text { Arthropods } \\
\left(\text { low }-\mathrm{C}_{3}\right)\end{array}$ & $\begin{array}{l}\text { Arthropods } \\
\left(\text { high }-\mathrm{C}_{4}\right)\end{array}$ & $\begin{array}{l}\text { Arthropods } \\
\left(\text { low }-\mathrm{C}_{4}\right)\end{array}$ \\
\hline
\end{tabular}

\section{American Robin}

winter

$7 / 7$

96.5

31.0

69.0

$(89.5,100+) \quad(9.8,49.8) \quad(50.3,90.2)$

Dark-eyed Junco

winter

$12 / 12$

0

58.2

41.8

3.3

$(32.6,81.6) \quad(18.4,67.4)$

$(0.3,15.1)$

$(3.8,37.9)$

4.0

25.6

17.6

24.5

$(0.3,19.0) \quad(7.5,46.8) \quad(2.4,35.2)$

$(6.3,42.2)$

Eastern Towhee

fall

$10 / 6$

29.2

22.8

48.6

28.4

18.8

14.2

$(0,76.8)$

(7.1, 37.6)

$(30.2,66.4)(10.9,47.9)$

$(5.0,31.8)$

$(2.1,28.8)$

7.7

5.9

21.5

6.4

Gray Catbird

fall

$10 / 6$

57.5
$(22.7,92.3)$

16.9

83.1

$(2.9,32.0) \quad(68.0,97.1)$
41.3

41.4

$(29.8,53.5) \quad(28.2,55.3)$

Hermit Thrush 
winter

$\begin{array}{cccc}11 / 11 & 71.4 & 9.0 & 91.1 \\ & (48.6,94.1) & (0.9,28.6) & (71.4,99.1)\end{array}$
$29.4 \quad 60.0$

$(16.3,44.2) \quad(41.5,71.7)$

Northern Cardinal

fall

winter

Ruby-crowned Kinglet

fall

winter

Swainson's Thrush

fall
23.4 $(11.4,37.9) \quad(37.5,73.7)$

Tufted Titmouse

fall

34.0
$(0,91.9)$

\author{
25.8
}

45.8

27.5

5.7

24.5

3.4

8.2

20.5

6.0

$11 / 4 \quad 48.8$

$(0,100+) \quad(2.3,32.1) \quad(26.9,74.1) \quad(13.2,54.7)$

3.7

20.9

$(0.3,11.7)$

$(0.9,19.9)$

$(3.3,42.7)$

$(0.7,15.8)$

$(0.3,17.1) \quad(4.8,38.5)$

\begin{abstract}
3.8
\end{abstract}
20.9

14.1

17.8

$(3.4,33.5)$ 
White-throated Sparrow

\begin{tabular}{|c|c|c|c|c|c|c|c|c|c|c|c|}
\hline fall & $5 / 11$ & 0 & - & $\begin{array}{c}67.6 \\
(47.2,87.7)\end{array}$ & $\begin{array}{c}32.4 \\
(12.3,52.8)\end{array}$ & $\begin{array}{c}19.2 \\
(3.2,34.1)\end{array}$ & $\begin{array}{c}20.6 \\
(4.4,36.2)\end{array}$ & $\begin{array}{c}12.7 \\
(1.3,28.2)\end{array}$ & $\begin{array}{c}14.2 \\
(1.7,29.8)\end{array}$ & $\begin{array}{c}20.1 \\
(4.0,35.1)\end{array}$ & $\begin{array}{c}11.9 \\
(1.3,27.3)\end{array}$ \\
\hline winter & $5 / 0$ & - & - & $\begin{array}{c}64.4 \\
(41.7,85.8)\end{array}$ & $\begin{array}{c}35.6 \\
(14.3,58.3)\end{array}$ & $\begin{array}{c}10.4 \\
(0.9,27.2)\end{array}$ & $\begin{array}{c}20.5 \\
(4.3,37.1)\end{array}$ & $\begin{array}{c}10.0 \\
(0.8,27.2)\end{array}$ & $\begin{array}{c}20.1 \\
(3.7,36.7)\end{array}$ & $\begin{array}{c}18.7 \\
(3.1,34.5)\end{array}$ & $\begin{array}{c}17.2 \\
(2.7,33.2)\end{array}$ \\
\hline
\end{tabular}

Yellow-rumped Warbler

\begin{tabular}{|c|c|c|c|c|c|c|c|c|c|c|c|}
\hline Fall 1998 \& 1999 & $9 / 32$ & $\begin{array}{c}77.3 \\
(68.0,86.6)\end{array}$ & $\begin{array}{c}10.2 \\
(1.2,25.1)\end{array}$ & $\begin{array}{c}89.8 \\
(74.9,98.8)\end{array}$ & - & $\begin{array}{c}64.5 \\
(48.2,79.7)\end{array}$ & $\begin{array}{c}24.0 \\
(8.8,42.1)\end{array}$ & - & - & - & - \\
\hline winter $98 / 99$ & $17 / 34$ & $\begin{array}{c}72.5 \\
(65.9,79.1)\end{array}$ & $\begin{array}{c}36.8 \\
(24.2,47.6)\end{array}$ & $\begin{array}{c}63.2 \\
(52.4,75.8)\end{array}$ & - & $\begin{array}{c}1.2 \\
2(0.1,5.2)\end{array}$ & $\begin{array}{c}61.5 \\
(51.1,73.6)\end{array}$ & - & - & - & - \\
\hline winter $99 / 00$ & $12 / 37$ & $\begin{array}{c}92.9 \\
(76.2,100+)\end{array}$ & $\begin{array}{c}19.2 \\
(5.4,32.3)\end{array}$ & $\begin{array}{c}80.8 \\
(67.7,94.7)\end{array}$ & - & $\begin{array}{c}28.5 \\
(19.9,38.7)\end{array}$ & $\begin{array}{c}52.0 \\
(39.8,65.0)\end{array}$ & - & - & - & - \\
\hline
\end{tabular}


Table 5. Species specific probabilities that dietary end points differ among seasons. Probabilities are symmetrical around 0.5 , as a result, a probability of $0.75=$ a probability of 0.25 where a probability of 0.5 indicates no differences among seasons.

\begin{tabular}{|c|c|c|c|c|c|c|}
\hline Species & Comparison & Fruit & Arth & $\begin{array}{c}\text { Arth } \\
\text { (H-C3) }\end{array}$ & $\begin{array}{c}\text { Arth } \\
\text { (L-C3) }\end{array}$ & $\begin{array}{c}\text { Arth } \\
(\mathrm{H}-\mathrm{C} 4)\end{array}$ \\
\hline Northern Cardinal & Winter $>$ Fall? & 0.25 & 0.60 & 0.61 & 0.40 & 0.35 \\
\hline Ruby-crowned Kinglet & Winter $>$ Fall? & 0.84 & 0.16 & 0.49 & 0.21 & - \\
\hline Yellow-rumped Warbler & Winter $98>$ Fall? & 0.98 & 0.02 & 0.00 & 1.00 & - \\
\hline Yellow-rumped Warbler & Winter $99>$ Fall? & 0.77 & 0.23 & 0.00 & 0.98 & - \\
\hline Yellow-rumped Warbler & Winter $99>$ Winter $1998 ?$ & 0.06 & 0.94 & 1.00 & 0.17 & - \\
\hline White-throated Sparrow & Winter $>$ Fall? & - & 0.43 & 0.28 & 0.50 & 0.43 \\
\hline
\end{tabular}




\section{Figure Legends}

Figure 1. $\delta^{15} \mathrm{~N}$ and $\delta^{13} \mathrm{C}$ Isotopic signature of bird feathers (A) which were grown during the late summer months and consequently, influenced by late summer diet and blood (ruby-crowned kinglet only) and plasma (all other species) collected during the fall (B) and winter (C) months. When comparing the isotopic signatures of these tissues, it appears that there is broad overlap in diet during the summer and that diet becomes increasingly differentiated among species in the fall and especially during the winter (although we recommend caution comparing isotopic signatures among tissues because of differences in discrimination factors). The dietary end point graph (D) is provided as a reference and, when compared to the isotopic signatures of birds in the winter, this comparison suggests that birds increase the use of fruit (less enriched $\mathrm{N}$ values in the winter) and seeds (more enriched $\mathrm{C}$ values during the winter). 
Figure 1

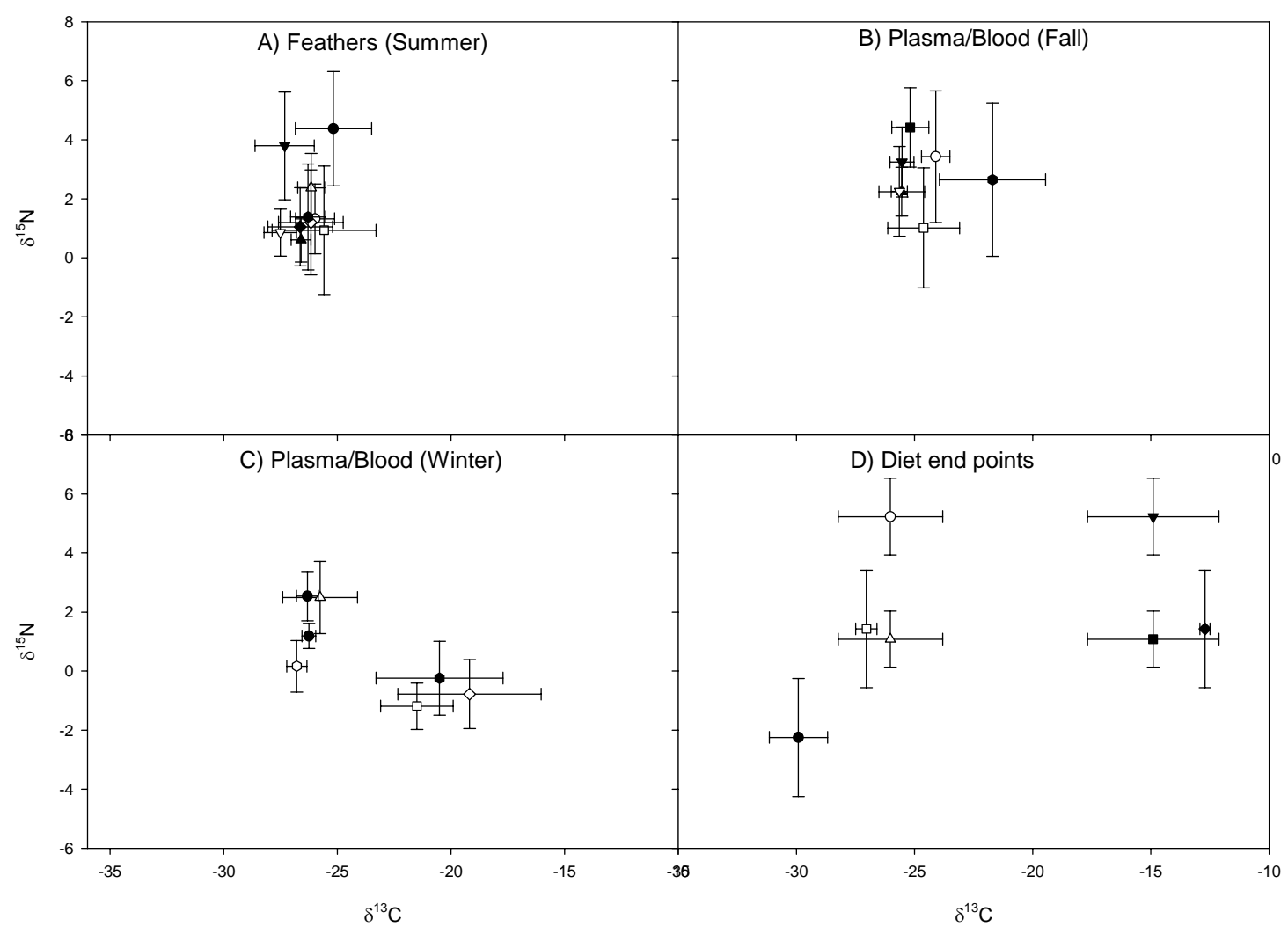

Bird Tissue Graphs (A-C)

- American Robin

- Eastern Towhee

$\checkmark$ Gray Catbird

$\triangle$ Hermit Thrush

- Yellow-rumped Warbler

- Northern Cardinal

- Ruby-crowned Kinglet

$\diamond \quad$ Dark-eyed Junco

- Swainson's Thrush

$\nabla$ Tufted Titmouse

- White-throated Sparrow

- Yellow-rumped Warbler (Winter 1998)

- Yellow-rumped Warbler (Winter 1999)

- Fruits

- High Trophic Arthropods (C3)

7 High Trophic Arthropods (C4)

$\triangle \quad$ Low Trophic Arthropods (C3)

- Low Trophic Arthropods (C4)

- Seeds (C3) 